\title{
THE DYNAMIC PROGRAMMING EQUATION FOR THE PROBLEM OF OPTIMAL INVESTMENT UNDER CAPITAL GAINS TAXES*
}

\author{
IMEN BEN TAHAR ${ }^{\dagger}$, H. METE SONER $\ddagger$, AND NIZAR TOUZI $\S$
}

\begin{abstract}
This paper considers an extension of the Merton optimal investment problem to the case where the risky asset is subject to transaction costs and capital gains taxes. We derive the dynamic programming equation in the sense of constrained viscosity solutions. We next introduce a family of functions $\left(V_{\varepsilon}\right)_{\varepsilon>0}$, which converges to our value function uniformly on compact subsets, and which is characterized as the unique constrained viscosity solution of an approximation of our dynamic programming equation. In particular, this result justifies the numerical results reported in the accompanying paper [5].
\end{abstract}

Key words. Optimal consumption and investment in continuous-time, transaction costs, capital gains taxes, viscosity solutions.

AMS subject classifications. 91B28, 49J20, 35D99.

1. Introduction. The problem of optimal investment and consumption in financial markets has been introduced by Merton [20, 21]. The explicit solution derived in these papers is widely used among fund managers in practical financial markets. Moreover, this problem became very quickly one of the classical examples of application of the verification theorem in stochastic control theory. Indeed, by direct financial considerations, it is easily seen that the value function of the problem satisfies some homogeneity property, which completely determines its dependence on the wealth state variable. Plugging this information into the corresponding dynamic programming equation (DPE) leads to an ordinary differential equation which can be solved explicitly, thus providing a candidate smooth solution to the DPE.

In this paper, we consider the extension of the Merton problem to the case where the risky asset is subject to capital gains taxes. For technical reasons, we also assume that the risky asset is subject to proportional transaction costs. This problem is formulated in the accompanying paper [5]. In contrast with the Merton frictionless model, no explicit solution is available in this context. The main result of [5] is the derivation of an explicit first order expansion of the value function for small tax and interest rate parameters. The numerical results reported in [5] show that the relative error induced by this approximation is of the order of $4 \%$. These numerical results are obtained by comparing the explicit first order expansion to the finite differences approximation of the solution of the corresponding DPE.

The literature on the optimal investment problem under capital gains taxes is not very expanded, and is mainly developed in discrete-time binomial models, see [7], [10], [11], [12], [17, 18], [15] and [19].

The main purpose of this paper is to justify the approximation of the value function by means of the finite differences scheme applied to the corresponding DPE. Since

\footnotetext{
*Authors would like to thank anonymous referees for insightful comments.

† CEREMADE, Université Paris Dauphine, France, bentahar@ceremade.dauphine.fr.

$\ddagger$ Koç University, Istanbul, Turkey, msoner@ku.edu.tr. Member of the Turkish Academy of Sciences and this work was partly supported by the Turkish Academy of Sciences and by the Turkish Scientific and Technological Research Institute, TÜBITAK.

${ }^{\S}$ Centre de Mathématiques Appliquées, Ecole Polytechnique Paris, France, touzi@cmap.polytechnique.fr, and Tanaka Business School, Imperial College London, UK, n.touzi@imperial.ac.uk.
} 
our optimal control problem is singular, the DPE takes the form of a variational inequality :

$$
\min \left\{-\mathcal{L} v, \mathbf{g}^{\mathbf{b}} \cdot D v, \mathbf{g}^{\mathbf{s}} \cdot D v\right\}=0 \text { on } \overline{\mathcal{S}}, v=0 \text { on } \partial^{z} \mathcal{S}
$$

where $\mathcal{L}$ is a second order differential operator defined in $(2.13), \mathbf{g}^{\mathbf{b}}, \mathbf{g}^{\mathbf{s}}$ are two vector fields defined in $(2.15)$, corresponding to the purchase and sale decisions, $\mathcal{S}$ is the state space, and $\partial^{z} \mathcal{S}$ is part of the boundary of $\mathcal{S}$. The main difficulty comes from the fact that the vector field $\mathbf{g}^{\mathbf{s}}$ is not locally Lipschitz. Then the standard techniques to prove a uniqueness result for the above PDE fail. We then introduce a convenient locally Lipschitz approximation $\mathbf{g}_{\varepsilon}^{\mathbf{s}}$ of $\mathbf{g}^{\mathbf{s}}$, and we consider the approximating PDE

$$
\min \left\{-\mathcal{L} v, \mathbf{g}^{\mathbf{b}} \cdot D v, \mathbf{g}_{\varepsilon}^{\mathbf{s}} \cdot D v\right\}=0 \text { on } \overline{\mathcal{S}}, v=0 \text { on } \partial^{z} \mathcal{S}
$$

The main result of this paper states that the above approximating PDE has a unique continuous viscosity solution $V_{\varepsilon}$ which converges uniformly on compact subsets towards the value function $V$ of our optimal investment problem under capital gains taxes. Applying the general results of Barles and Souganidis [4], this justifies the convergence of the numerical scheme implemented in the accompanying paper [5] towards this unique solution of the approximating PDE.

The paper is organized as follows. Section 2 provides a quick review of the problem of optimal investment under capital gains taxes. The main approximation result is stated in Section 3. In Section 4, we prove a comparison result for the approximating $\mathrm{PDE}$, which implies the required uniqueness claim. The existence of a solution of the approximating PDE is proved in Section 5 by introducing a family of control problems obtained by modifying conveniently our original problem. Finally, Section 6 reports the proof of convergence of $V_{\varepsilon}$ towards $V$ uniformly on compact subsets.

Notations : For a domain $\mathbf{D}$ in $\mathbb{R}^{n}$, we denote by $\operatorname{USC}(\mathbf{D})$ (resp. $\operatorname{LSC}(\mathbf{D})$ ) the collection of all upper semi-continuous (resp. lower semi-continuous) functions from $\mathbf{D}$ to $\mathbb{R}$. The set of continuous functions from $\mathbf{D}$ to $\mathbb{R}$ is denoted by $\mathrm{C}^{0}(\mathbf{D}):=$ $\mathrm{USC}(\mathbf{D}) \cap \operatorname{LSC}(\mathbf{D})$. For a parameter $\delta>0$, we say that a function $f: \mathbf{D} \longrightarrow \mathbb{R}$ has $\delta$-polynomial growth if

$$
\sup _{x \in \mathbf{D}} \frac{|f(x)|}{1+|x|^{\delta}}<\infty .
$$

We finally denote by $\mathrm{USC}_{\delta}(\mathbf{D}):=\{f \in \mathrm{USC}(\mathbf{D}): f$ has $\delta$-polynomial growth $\}$. The sets $\operatorname{LSC}_{\delta}(\mathbf{D})$ and $\mathrm{C}_{\delta}^{0}(\mathbf{D})$ are defined similarly.

\section{Optimal investment under capital gains taxes.}

2.1. Problem formulation. In this section, we review quickly the formulation of the problem of optimal investment under capital gains taxes. We refer the interested reader to the accompanying paper [5] for more details. The financial market consists of a tax-free bank account with constant interest rate $r>0$, and a risky asset subject to proportional transaction costs and to capital gains taxes. The price process of the risky asset evolves according to the Black and Scholes model :

$$
d P_{t}=P_{t}\left(\rho d t+\sigma d W_{t}\right), t \geq 0
$$

where $\rho>0$ is a constant instantaneous return of the asset, and $\sigma>0$ is a constant volatility parameter. The process $W=\left\{W_{t}, t \geq 0\right\}$ is a standard Brownian motion 
with values in $\mathbb{R}^{1}$ defined on an underlying complete probability space $(\Omega, \mathcal{F}, \mathbb{P})$. We shall denote by $\mathbb{F}$ the $\mathbb{P}$-completion of the natural filtration of the Brownian motion.

For technical reasons, see Section 4, we assume that the risky asset is also subject to proportional transaction costs defined by the coefficients $\lambda, \mu \in[0,1)$, so that the bid and ask prices at time $t$ of the risky asset are given by $(1-\mu) P_{t}$ and $(1+\lambda) P_{t}$.

A control process is a triple of $\mathbb{F}$-adapted processes $\nu=(C, L, M)$ where

$$
C \geq 0 \text { and } \int_{0}^{T} C_{t} d t<\infty \mathbb{P}-\text { a.s. for all } T>0,
$$

$L$, and $M$ are non-decreasing right-continuous, $L_{0-}=M_{0-}=0$, and the jumps of $M$ satisfy

$$
\Delta M_{t} \leq 1 \text { fort } \geq 0 \mathbb{P}-\text { a.s. }
$$

Here $C_{t}$ is the consumption rate at time $t, d L_{t} \geq 0$ is the amount invested between times $t$ and $t+d t$ to purchase risky assets, and $d M_{t} \geq 0$ is the proportion of risky assets in portfolio which is sold between times $t$ and $t+d t$. Then, the amount of wealth $Y=\left\{Y_{t}, t \geq 0\right\}$ on the risky asset account is defined by the dynamics

$$
d Y_{t}=Y_{t} \frac{d P_{t}}{P_{t}}+d L_{t}-Y_{t-} d M_{t}, \quad t \geq 0 .
$$

Since $\Delta M_{t} \leq 1$, the no short-sales constraint $Y \geq 0$ holds. Capital gains are taxed only when the investor sells the risky asset. The amount of capital gains (or losses) is evaluated by comparing the actual price $P_{t}$ to a tax basis $B_{t}$ specified by the taxation code. In our framework the tax basis is defined as the weighted average of past purchase prices

$$
B_{t}:=\frac{K_{t}}{Y_{t}} P_{t} \text { if } Y_{t}>0 \text { and } B_{t}:=P_{t} \text { otherwise }, t \geq 0,
$$

where

$$
d K_{t}=d L_{t}-K_{t-} d M_{t}, t \geq 0 .
$$

The natural initial condition for the process $K$ is zero as initially there are no prior stocks bought. However, the method of dynamic programming always forces us to consider all possible initial data. Hence we consider the $K$-equation with a general initial data $K_{0}=k$. Also a more detailed derivation of this tax basis and its place in actual tax codes is given in Subsection 2.2 of the accompanying paper [5].

Finally, we consider a linear taxation rule, with constant tax rate parameter $\alpha \in[0,1]$, so that the after-tax and after-transaction costs amount induced by selling the amount $Y_{t-} d M_{t}$ between times $t$ and $t+d t$ is given by

$(1-\mu) Y_{t-} d M_{t}-\alpha(1-\mu)\left[Y_{t-} d M_{t}-\frac{Y_{t-} d M_{t}}{P_{t}} B_{t-}\right]=(1-\mu)\left[(1-\alpha) Y_{t-}+\alpha K_{t-}\right] d M_{t}$.

This justifies the following dynamics for the non-risky asset component of wealth process :

$$
d X_{t}=\left(r X_{t}-C_{t}\right) d t-(1+\lambda) d L_{t}+(1-\mu)\left[(1-\alpha) Y_{t-}+\alpha K_{t-}\right] d M_{t}, t \geq 0(.2 .6
$$


We denote by $\mathcal{A}$ the set of all control processes, and by $S=(X, Y, K)$ the corresponding state process defined by (2.4)-(2.5)-(2.6). A control process $\nu$ is said to be admissible if the no bankruptcy condition

$$
Z_{t}:=X_{t}+(1-\mu)\left[(1-\alpha) Y_{t}+\alpha K_{t}\right] \geq 0, t \geq 0 \mathbb{P}-\text { a.s. }
$$

holds. Here $Z_{t}$ is the after-tax and after transaction-costs liquidation value of the portfolio at time $t$. Given an initial condition $S_{0_{-}}=s$, we shall denote by $\mathcal{A}(s)$ the collection of all admissible controls.

The problem of optimal consumption and investment under capital gains taxes is defined by the value function

$$
V(s):=\sup _{\nu \in \mathcal{A}(s)} \mathbb{E}\left[\int_{0}^{\infty} e^{-\beta t} U\left(C_{t}\right) d t\right], \text { where } U(x):=\frac{x^{p}}{p}, x \geq 0,
$$

and $\beta>0, p \in(0,1)$ are two given constant parameters.

Throughout this paper, we assume that the coefficients of the model satisfy the condition

$$
\frac{\beta}{p}-r-\frac{(\delta-r)^{2}}{2(1-p) \sigma^{2}}>0
$$

which ensures that the value function of the Merton optimal consumption-investment problem (the case $\lambda=\mu=\alpha=0$ ) is finite. In particular, the value function $V$ is finite under Condition (2.9).

2.2. The dynamic programming equation. For an admissible control $\nu \in$ $\mathcal{A}(s)$, the induced state process $S^{\nu}=\left(X^{\nu}, Y^{\nu}, K^{\nu}\right)$ defined by (2.4)-(2.5)-(2.6) together with some initial data $S_{0}^{\nu}=s$ is valued in the state space

$$
\overline{\mathcal{S}}:=\left\{(x, y, k) \in \mathbb{R} \times \mathbb{R}_{+} \times \mathbb{R}_{+}: z=x+(1-\mu)[(1-\alpha) y+\alpha k] \geq 0\right\}
$$

We denote by $\mathcal{S}:=\operatorname{int}(\overline{\mathcal{S}})$ the interior of $\overline{\mathcal{S}}$, and we decompose the boundary of this state space into $\partial \mathcal{S}=\partial^{y} \mathcal{S} \cup \partial^{k} \mathcal{S} \cup \partial^{z} \mathcal{S}$, where

$$
\partial^{y} \mathcal{S}=\{s \in \mathcal{S}: y=0\}, \partial^{k} \mathcal{S}=\{s \in \mathcal{S}: k=0\} \text { and } \partial^{z} \mathcal{S}=\{s \in \mathcal{S}: z=0\} .
$$

Observe that the value function is not known on all of the boundary of the state space $\mathcal{S}$. It is shown in [5] that the only boundary information is

$$
V(s)=0 \text { for all } s \in \partial^{z} \mathcal{S} .
$$

The main result of this section states that the value function $V$ defined in (2.8) solves the corresponding DPE

$$
F\left(s, v, D v, D^{2} v\right):=\min \left\{-\mathcal{L} v, \mathbf{g}^{\mathbf{b}} \cdot D v, \mathbf{g}^{\mathbf{s}} \cdot D v\right\}=0 \mathrm{on} \overline{\mathcal{S}} \backslash \partial^{z} \mathcal{S},
$$

where $\mathcal{L}$ is the second order differential operator

$$
\mathcal{L} \varphi(s):=-\beta \varphi(s)+r x \varphi_{x}(s)+\rho y \varphi_{y}(s)+\frac{1}{2} \sigma^{2} y^{2} \varphi_{y y}(s)+\tilde{U}\left(\varphi_{x}(s)\right),
$$

$\tilde{U}$ is the Fenchel dual defined by

$$
\tilde{U}(\xi):=\sup _{c>0}(U(c)-c \xi) \text { for all } \xi>0,
$$


and $\mathbf{g}^{\mathbf{b}}, \mathbf{g}^{\mathbf{s}}$ are the vector fields defined by

$$
\mathbf{g}^{\mathbf{b}}:=\left(\begin{array}{c}
1+\lambda \\
-1 \\
-1
\end{array}\right), \mathbf{g}^{\mathbf{s}}(s):=\left(\begin{array}{c}
-(1-\mu) \\
\frac{1}{1-\alpha} \\
0
\end{array}\right)+\left(\begin{array}{c}
0 \\
\frac{-\alpha}{1-\alpha} \\
1
\end{array}\right) \frac{k \mathbf{1}_{(y, k) \neq 0}}{(1-\alpha) y+\alpha k} .(2
$$

The dynamic programming equation can be written in different forms by taking other vector fields which are parallel to our choices $\mathrm{g}^{\mathbf{b}}, \mathrm{g}^{\mathbf{s}}$. Since, our choice for $\mathrm{g}^{\mathbf{s}}$ is discontinuous and this fact is central to many of the technicalities, one may propose to choose a parallel vector field which is continuous. However, in singular stochastic control, if the vector fields appearing in the equation vanishes (which is the case here if we choose continuous vector fields) then the first order part of the equation (i.e., the part $\mathbf{g}^{\mathbf{s}} \cdot D v$ in the above particular case) becomes degenerate. Indeed, this degeneracy is equivalent to the technical difficulties related to the discontinuity of the vector fields. For this reason, it is standard in singular control to choose these vector fields non-degenerate and close to unit vector fields.

Since we have no knowledge of any a priori regularity of the value function $V$, we will use the theory of viscosity solutions. This notion allows for a weak formulation of solutions to second order parabolic partial differential equations and boundary conditions, see [23], [9].

In the sequel, we shall make use of the following classical notations in the viscosity theory. For a locally bounded function $v: \overline{\mathcal{S}} \longrightarrow \mathbb{R}$, we denote the corresponding upper and lower semi-continuous envelopes by

$$
v^{*}(s):=\limsup _{\mathcal{S} \ni s^{\prime} \rightarrow s} v\left(s^{\prime}\right) \text { and } v_{*}(s):=\liminf _{\mathcal{S} \ni s^{\prime} \rightarrow s} v\left(s^{\prime}\right) .
$$

The notation $F_{*}$ in the subsequent definition is defined similarly. Observe that $F=F_{*}$ outside the axis $\{(x, 0,0): x \geq 0\}$.

Definition 2.1. (i) A locally bounded function $v$ is a constrained viscosity subsolution of (2.11)-(2.12) if $v^{*} \leq 0$ on $\partial^{z} \mathcal{S}$, and for all $s \in \overline{\mathcal{S}} \backslash \partial^{z} \mathcal{S}$ and $\varphi \in C^{2}(\overline{\mathcal{S}})$ with $\left(v^{*}-\varphi\right)(s)=\max _{\overline{\mathcal{S}}}\left(v^{*}-\varphi\right)$ we have $F_{*}\left(s, v(s), D \varphi(s), D^{2} \varphi(s)\right) \leq 0$.

(ii) A locally bounded function $v$ is a viscosity supersolution of (2.11)-(2.12) if $v_{*} \geq 0$ on $\partial^{z} \mathcal{S}$, and for all $s \in \mathcal{S}$ and $\varphi \in C^{2}(\mathcal{S})$ with $\left(v_{*}-\varphi\right)(s)=\min _{\mathcal{S}}\left(v_{*}-\varphi\right)$ we have $F\left(s, v(s), D \varphi(s), D^{2} \varphi(s)\right) \geq 0$.

(iii) A locally bounded function $v$ is a constrained viscosity solution of (2.11)-(2.12) if it is a constrained viscosity subsolution and supersolution.

In the above definition, Observe that there is no boundary value assigned to the value function on $\partial^{y} \mathcal{S} \cup \partial^{k} \mathcal{S}$. Instead, the subsolution property holds on this boundary. Notice that the supersolution property is satisfied only in the interior of the domain $\mathcal{S}$.

Proposition 2.2. The value function $V$ is a constrained viscosity solution of (2.11)-(2.12)

The proof is reported in Section 5 in the case $\varepsilon=0$. In the accompanying paper [5] a numerical scheme based on the finite differences approximation of the DPE (2.11)-(2.12) is implemented. In order to justify this algorithm, a uniqueness result for this DPE is needed. As it is usually the case for parabolic second order equations, uniqueness follows as a consequence of a comparison result. At this point, a chief difficulty is encountered : the vector field $\mathbf{g}^{\mathbf{s}}$ is not locally Lipschitz on the axis $\{(x, 0,0), x \geq 0\}$. Because of this problem, the standard techniques for the derivation of a comparison result for the DPE (2.11)-(2.12) fail. 
REMARK 1. Consider the Lipschitz vector field $\mathbf{G}^{\mathbf{s}}:=(-(1-\mu)[(1-\alpha) y+\alpha k], y, k)=$ $[(1-\alpha) y+\alpha k] \mathbf{g}^{\mathbf{s}}$. Then, the supersolutions of (2.11)-(2.12) coincide with those of

$$
\min \left\{-\mathcal{L} v, \mathbf{g}^{\mathbf{b}} \cdot D v, \mathbf{G}^{\mathbf{s}} \cdot D v\right\} \geq 0 \text { on } \overline{\mathcal{S}} \backslash \partial^{z} \mathcal{S} \text { and } v=0 \text { on } \partial^{z} \mathcal{S} .
$$

However these two equations do not have the same set of subsolutions. The reason for this is that the subsolution property must hold also on the boundary $\partial^{y} \mathcal{S} \cup \partial^{k} \mathcal{S}$. Since $\mathbf{G}^{\mathbf{s}}(x, 0,0)=0$ for every $x \geq 0$, the equation (2.16) provides no information on this axis. Notice however that $\lim _{n \rightarrow \infty} \mathbf{g}^{\mathbf{s}}\left(s_{n}\right)$ exists for some sequences $s_{n} \rightarrow(x, 0,0)$, and might be non-zero, so that equation (2.12) bears more information on this axis.

This remark justifies that the above mentioned difficulty can be avoided if a priori comparison on the axis $\{(x, 0,0): x \geq 0\}$ were available.

Proposition 2.3. Let $\lambda+\mu>0$. Let $u$ be an upper-semicontinuous constrained viscosity subsolution of (2.11)-(2.12), and $v$ be a lower-semicontinuous viscosity supersolution of (2.11)-(2.12) with $(u-v)^{+} \in \mathrm{USC}_{p}(\overline{\mathcal{S}})$. Assume further that $(u-v)(x, 0,0) \leq 0$ for all $x \geq 0$. Then $u \leq v$ on $\overline{\mathcal{S}}$.

The proof of this comparison result is given at the end of Section 4. Unfortunately, this result does not provide uniqueness of a constrained viscosity solution for the DPE (2.11)-(2.12), as we have no a priori comparison of two possible solutions on the axis $\{(x, 0,0): x \geq 0\}$.

The chief goal of this paper is to obtain an alternative characterization of $V$ by considering a convenient approximating PDE which has a unique solution converging to our value function $V$. Before turning to this issue, we report the following continuity property from [5] which follows from Proposition 2.3.

Proposition $2.4([5])$. Let $\lambda+\mu>0$. For $s=(x, y, k) \in \overline{\mathcal{S}}$ and $z:=x+(1-$ $\mu)[(1-\alpha) y+\alpha k]$, we have $V(s)=z^{p} \mathcal{V}\left(\frac{y}{z}, \frac{k}{z}\right)$, where $\mathcal{V}$ is a Lipschitz-continuous function on $\mathbb{R}_{+}^{2}$.

3. The main results. For every $\varepsilon>0$ and $s=(x, y, k) \in \overline{\mathcal{S}}$, we define

$$
f^{\varepsilon}(s):=h\left(\frac{k}{\varepsilon z}\right)^{+} \text {where } z:=x+(1-\mu)[(1-\alpha) y+\alpha k]
$$

and $h$ is a non-decreasing $C^{2}\left(\mathbb{R}_{+}\right)$-function with

$$
h=0 \text { on }[0,1] \text { and } h=1 \text { on }[2, \infty) .
$$

For $\varepsilon=0$, we set $f^{0}(s)=1$.

We next introduce, for all $\varepsilon \geq 0$, the approximation $\mathbf{g}_{\varepsilon}^{\mathbf{s}}$ of $\mathbf{g}^{\mathbf{s}}$ :

$$
\mathbf{g}_{\varepsilon}^{\mathbf{s}}(s):=\mathbf{g}^{\mathbf{s}}\left(x, y, k f^{\varepsilon}(s)\right) \text { for } s=(x, y, k) \in \overline{\mathcal{S}},
$$

and the corresponding approximation of the DPE (2.11)-(2.12) :

$$
\min \left\{-\mathcal{L} v, \mathbf{g}^{\mathbf{b}} \cdot D v, \mathbf{g}_{\varepsilon}^{\mathbf{s}} \cdot D v\right\}=0 \text { on } \overline{\mathcal{S}} \backslash \partial^{z} \mathcal{S} \text { and } v=0 \text { on } \partial^{z} \mathcal{S} .
$$

A constrained viscosity solution of this equation is defined exactly as in definition 2.1, replacing $\mathbf{g}^{\mathbf{s}}$ by $\mathbf{g}_{\varepsilon}^{\mathbf{s}}$. For each $\varepsilon>0$ the approximation $\mathbf{g}_{\varepsilon}^{\mathbf{s}}$ is Lipschitz-continuous on $\overline{\mathcal{S}} \backslash \partial^{z} \mathcal{S}$, and this property is sufficient to obtain the following comparison result.

ThEOREM 3.1. Let $\lambda+\mu>0$ and $\varepsilon>0$. Let $u$ be an upper-semicontinuous constrained viscosity subsolution of (3.3), and $v$ be a lower-semicontinuous viscosity 
supersolution of $(3.3)$ with $(u-v)^{+} \in \operatorname{USC}_{p}(\overline{\mathcal{S}})$. Assume further that $u \leq v$ on $\partial^{z} \mathcal{S}$. Then $u \leq v$ on $\overline{\mathcal{S}}$.

This result is proved in Section 4, and implies as usual a uniqueness result for the approximating PDE (3.3) for every $\varepsilon>0$. We can now state our main DPE characterization of the value function $V$ which justifies the numerical scheme implemented in the accompanying paper [5].

THEOREM 3.2. For every $\varepsilon>0$, there exists a unique constrained viscosity solution $V_{\varepsilon}$ for the nonlinear parabolic PDE (3.3) in the class $\mathrm{C}_{p}^{0}$. Moreover, the family $\left(V_{\varepsilon}\right)_{\varepsilon>0}$ is non-decreasing and converges to the value function $V$ uniformly on compact subsets of $\overline{\mathcal{S}}$ as $\varepsilon \backslash 0$.

The existence of a solution for the approximating PDE (3.3) is proved in Section 5 by conveniently modifying the optimal investment problem under capital gains taxes, and showing that the induced value function $V_{\varepsilon}$ is a constrained viscosity solution of (3.3). Moreover, we will prove in Proposition 6.2 that $0 \leq V_{\varepsilon} \leq V$, so that $V_{\varepsilon}$ inherits the $p$-polynomial growth of $V$ stated in [5]. Together with the comparison result of Theorem (3.1), this shows that $V^{\varepsilon}$ is the unique constrained viscosity solution in $C_{p}^{0}$. The convergence result is proved in Section 6 .

4. The comparison result. We adapt the standard argument based on the Ishii technique, see Theorem 3.2 and Lemma 3.1 in [9]. The subsequent proof is also inspired from [1]. In comparison to the latter paper, we have the additional difficulty implied by the state constraint $(y, k) \in \mathbb{R}_{+}^{2}$. We use the idea of Theorem 7.9 in [9] to account for this avoiding the continuity assumptions of this theorem. We mention that comparison results for second order PDE's with state constraints have been obtained for specific control problems in [2] and [3], but do not apply to our context. In the subsequent analysis, the key-result to avoid the continuity is the observation that

$$
\begin{gathered}
\text { for each } s \in \overline{\mathcal{S}} \backslash \partial^{z} \mathcal{S}, \text { there exists some } \zeta_{s}>0 \text { such that } \\
\qquad-\zeta \mathbf{g}^{\mathbf{b}} \in \mathcal{S} \text { for every } 0<\zeta<\zeta_{s},
\end{gathered}
$$

together with the following

LEMma 4.1. Let $v \in \operatorname{LSC}(\overline{\mathcal{S}})$ be such that $v\left(s_{0}\right)=\liminf _{\mathcal{S} \ni s \rightarrow s_{0}} v(s)$, for $s_{0} \in \partial \mathcal{S}$. Assume that $\mathbf{g}^{\mathbf{b}} \cdot D v \geq 0$ on $\mathcal{S}$ in the viscosity sense. Then

$$
\lim _{\ell \backslash 0} v\left(s-\ell \mathbf{g}^{\mathbf{b}}\right)=v(s) \text { for anys } \in \overline{\mathcal{S}} \backslash \partial^{z} \mathcal{S} .
$$

Proof. Since $v$ is a viscosity super-solution of $\mathbf{g}^{\mathbf{b}} \cdot D v \geq 0$ on $\mathcal{S}$ and (4.1) holds, we deduce that, for any $s \in \mathcal{S}$, the function $\ell \longmapsto v\left(s-\ell \mathbf{g}^{\mathbf{b}}\right)$ is well defined and non-increasing on a neighborhood of 0 . In particular, $v\left(s-\ell \mathbf{g}^{\mathbf{b}}\right) \leq v(s)$ for any $s \in \mathcal{S}$, and $\ell \geq 0$ sufficiently small. For $s_{0} \in \partial \mathcal{S}$, it follows from the assumption of the lemma that $v\left(s_{0}\right)=\liminf _{\mathcal{S}_{\ni} \rightarrow s_{0}} v(s) \geq \liminf _{\mathcal{S}_{\mathcal{B}} \rightarrow s_{0}} v\left(s^{\prime}-\ell \mathbf{g}^{\mathbf{b}}\right) \geq v\left(s_{0}-\ell \mathbf{g}^{\mathbf{b}}\right)$. Hence

$$
v\left(s-\ell \mathbf{g}^{\mathbf{b}}\right) \leq v(s) \text { for any } s \in \overline{\mathcal{S}} \backslash \partial^{z} \mathcal{S} \text { and } \ell \geq 0 .
$$

This implies that, for any $s \in \overline{\mathcal{S}} \backslash \partial^{z} \mathcal{S}$,

$$
v(s) \geq \limsup _{\ell \searrow 0} v\left(s-\ell \mathbf{g}^{\mathbf{b}}\right) \geq \liminf _{\ell \searrow 0} v\left(s-\ell \mathbf{g}^{\mathbf{b}}\right) \geq \liminf _{\mathcal{S} \ni s^{\prime} \rightarrow s} v\left(s^{\prime}\right) \geq v(s),
$$

completing the proof. 
Another important ingredient of our comparison result is the use of a strict supersolution of the equation

$$
\min \left\{\mathbf{g}^{\mathbf{b}} \cdot D v, \mathbf{g}_{\varepsilon}^{\mathbf{s}} \cdot D v\right\}=0 \mathrm{on} \overline{\mathcal{S}} \backslash \partial^{z} \mathcal{S}
$$

This is the only place where the presence of transaction costs is crucial.

Lemma 4.2. Let $\lambda+\mu>0$ and assume that Condition (2.9) holds. Then, there exist two positive parameters

$$
0<\bar{\eta}<\frac{\lambda+\mu}{2} \text { and } \delta \in(p, 1) \text { with } \frac{\beta}{\delta}-r-\frac{\theta^{2}}{2(1-\delta)}>0,
$$

such that the function

$$
\Phi(s):=(x+(1-\mu)[(1-\alpha+\bar{\eta}) y+(\alpha+\bar{\eta}) k])^{\delta} \text { fors } \in \overline{\mathcal{S}}
$$

is a classical strict supersolution of (4.2).

Proof. We only show that $\mathbf{g}_{\varepsilon}^{\mathbf{s}} \cdot D \Phi>0$, as the other strict are easily seen to hold true. Setting $\tilde{z}:=x+(1-\mu)[(1-\alpha+\bar{\eta}) y+(\alpha+\bar{\eta}) k]$, we directly compute that

$$
\left(\mathbf{g}_{\varepsilon}^{\mathbf{s}} \cdot D \Phi\right)(s)=\frac{(1-\mu) \bar{\eta}}{1-\alpha} \tilde{z}^{\delta-1}\left[1+(1-2 \alpha) \frac{k f^{\varepsilon}(s)}{(1-\alpha) y+\alpha k f^{\varepsilon}(s)}\right]
$$

If $y=k=0$ or $1-2 \alpha \geq 0$, the required inequality is trivial. We next assume that $(y, k) \neq 0$ and $1-2 \alpha<0$. Then using the fact that $f^{\varepsilon}(s) \leq 1$, it follows that

$$
\begin{aligned}
\left(\mathbf{g}_{\varepsilon}^{\mathbf{s}} \cdot D \Phi\right)(s) & \geq \frac{(1-\mu) \bar{\eta}}{1-\alpha} \tilde{z}^{\delta-1}\left[1+(1-2 \alpha) \frac{k}{(1-\alpha) y+\alpha k)}\right] \\
& =\frac{(1-\mu) \bar{\eta}}{1-\alpha} \tilde{z}^{\delta-1} \frac{(1-\alpha)(y+k)}{(1-\alpha) y+\alpha k}>0 .
\end{aligned}
$$

We are now ready for the

Proof of Theorem 3.1 We start by setting a new notation. We denote by $\tilde{\mathcal{L}}$ the operator

$$
\tilde{\mathcal{L}}(s, u, q, Q):=-\beta u+r x q_{1}+\rho y q_{2}+\frac{1}{2} \sigma^{2} Q_{22}
$$

for $s=(x, y, k) \in \overline{\mathcal{S}}, u \in \mathbb{R}, q=\left(q_{i}\right)_{1 \leq i \leq 3} \in \mathbb{R}^{3}$, and $Q=\left(Q_{i, j}\right)_{\substack{1 \leq i \leq 3 \\ 1 \leq j \leq 3}} \in \mathbb{S}(3)$, so that the second order operator $\mathcal{L}$ can be written as

$$
\mathcal{L} \varphi(s)=\tilde{\mathcal{L}}\left(s, \varphi(s), D \varphi(s), D^{2} \varphi(s)\right)+\tilde{U}\left(\varphi_{x}(s)\right) .
$$

Let $u$ and $v$ be as in the statement of Theorem 3.1, and let us prove that $u \leq v$ in $\overline{\mathcal{S}}$.

We first observe that we can assume without loss of generality that

$$
v(s)=\liminf \left\{v\left(s^{\prime}\right): s^{\prime} \in \mathcal{S} \text { and } s^{\prime} \neq s\right\} \text { for everys } \in \partial^{y} \mathcal{S} \cup \partial^{k} \mathcal{S} .
$$

Indeed, we may define the function $\underline{v}:=v$ on $\mathcal{S} \cup \partial^{z} \mathcal{S}$ and $\underline{v}(s):=\liminf _{s \neq s^{\prime} \rightarrow s} v\left(s^{\prime}\right)$ for $s \in \partial^{y} \mathcal{S} \cup \partial^{k} \mathcal{S}$. Then, $\underline{v}$ satisfies the same conditions as $v$, and if we succeed in 
proving that $u \leq \underline{v}$, we deduce immediately that $u \leq v$ since the inequality $\underline{v} \leq v$ is trivial.

We now start the proof of the comparison result with the additional condition (4.3). Assume to the contrary that

$$
(u-v)\left(s^{*}\right)>0 \text { for somes } s^{*} \in \overline{\mathcal{S}} .
$$

1. Let $\Phi$ be the strict supersolution of (4.2) defined in Lemma 4.2, and $\eta>0, \zeta>0$ be some fixed parameters such that

$$
m_{0}:=(u-v)\left(s_{0}\right)-2 \eta \Phi\left(s_{0}\right)-\zeta\left|\mathbf{g}^{\mathbf{b}}\right|^{2}=\max _{s \in \overline{\mathcal{S}}}(u-v-2 \eta \Phi)-\zeta\left|\mathbf{g}^{\mathbf{b}}\right|^{2}>0
$$

by (4.4), where the maximum is attained thanks to the $p$-polynomial growth condition on $(u-v)^{+}$and the fact that $\delta>p$. In particular, it follows from (4.5) together with $\Phi \geq 0, u \leq v$ on $\partial^{z} \mathcal{S}$ and (4.1) that

$$
s_{0} \in \overline{\mathcal{S}} \backslash \partial^{z} \mathcal{S} \text { and } s_{0}-\zeta \mathbf{g}^{\mathbf{b}} \in \mathcal{S} \text { for small } \zeta>0 .
$$

We next define the mappings on $\overline{\mathcal{S}} \times \overline{\mathcal{S}}$ by

$$
\begin{aligned}
\Psi_{n}\left(s, s^{\prime}\right) & :=(u-\eta \Phi)(s)-(v+\eta \Phi)\left(s^{\prime}\right)-\psi_{n}\left(s, s^{\prime}\right), \\
\psi_{n}\left(s, s^{\prime}\right) & :=\left|n\left(s-s^{\prime}\right)-\zeta \mathbf{g}^{\mathbf{b}}\right|^{2}+\zeta\left|s-s_{0}\right|^{2} .
\end{aligned}
$$

Here, $\zeta \in(0,1)$ is some given constant. From the $p$-polynomial growth condition on $(u-v)^{+}$and the fact that $\delta>p$ in the definition of $\Phi$, we see that the uppersemicontinuous function $\Psi_{n}$ attains its maximum at some $\left(s_{n}, s_{n}^{\prime}\right)$ in $\overline{\mathcal{S}} \times \overline{\mathcal{S}}$, so that by $(4.5)$,

$$
m_{n}:=\Psi_{n}\left(s_{n}, s_{n}^{\prime}\right)=\max _{\left(s, s^{\prime}\right) \in \overline{\mathcal{S}} \times \overline{\mathcal{S}}} \Psi_{n}\left(s, s^{\prime}\right) \geq m_{0}>0
$$

By (4.6) and the definition of $\Psi_{n}$, we have the inequality $\Psi_{n}\left(s_{n}, s_{n}^{\prime}\right) \geq \Psi_{n}\left(s_{0}, s_{0}-\frac{\zeta}{n} \mathbf{g}^{\mathbf{b}}\right)$ which, together with the $p$-polynomial growth condition on $u$ and $v$ provides

$$
\begin{aligned}
\left|n\left(s_{n}-s_{n}^{\prime}\right)-\zeta \mathbf{g}^{\mathbf{b}}\right|^{2}+\zeta\left|s_{n}-s_{0}\right|^{2} \leq(u-\eta \Phi)\left(s_{n}\right)-(v+\eta \Phi)\left(s_{n}^{\prime}\right) & -(u-\eta \Phi)\left(s_{0}\right)+(v+\eta \Phi)\left(s_{0}-\frac{\zeta}{n} \mathbf{g}^{\mathbf{b}}(\hat{f}) \cdot 7\right) \\
& \leq \tilde{A}\left(1+\left|s_{n}\right|^{p}+\left|s_{n}^{\prime}\right|^{p}+\eta\left|s_{n}\right|^{\delta}+\eta\left|s_{n}^{\prime}\right|^{\delta}\right),
\end{aligned}
$$

for some positive constant $\tilde{A}$. We deduce from the last inequality that the sequences $\left(s_{n}\right)_{n \geq 1}$ and $\left(s_{n}^{\prime}\right)_{n \geq 1}$ are bounded, and we can assume without loss of generality that $s_{n}, s_{n}^{\prime} \longrightarrow \hat{s} \in \overline{\mathcal{S}}$ as $n \rightarrow \infty$. We now use Lemma 4.1, together with the uppersemicontinuity of $u$ and the lower-semicontinuity of $v$, to pass to the limit as $n \rightarrow \infty$ in (4.7). This provides :

$$
\begin{aligned}
\limsup _{n \rightarrow \infty}\left(\left|n\left(s_{n}-s_{n}^{\prime}\right)-\zeta \mathbf{g}^{\mathbf{b}}\right|^{2}+\zeta\left|s_{n}-s_{0}\right|^{2}\right) \leq & (u-\eta \Phi)(\hat{s})-(v+\eta \Phi)(\hat{s}) \\
& -\left((u-\eta \Phi)\left(s_{0}\right)-(v-\eta \Phi)\left(s_{0}\right)\right) \\
\leq 0 &
\end{aligned}
$$


where the last inequality follows from (4.5). Consequently

$$
\left|n\left(s_{n}-s_{n}^{\prime}\right)-\zeta \mathbf{g}^{\mathbf{b}}\right|^{2} \longrightarrow 0 \text { and } s_{n}, s_{n}^{\prime} \longrightarrow s_{0} \text { as } n \rightarrow \infty
$$

In particular, it follows from (4.6) that

$$
s_{n}^{\prime}=s_{n}-\frac{\zeta \mathbf{g}^{\mathbf{b}}+o(1)}{n} \in \mathcal{S} \text { and } s_{n} \in \overline{\mathcal{S}} \backslash \partial^{z} \mathcal{S} \text { for large } n .
$$

2. For each $n \geq 1,\left(s_{n}, s_{n}^{\prime}\right)$ is a maximum point of

$$
\Psi_{n}:\left(s, s^{\prime}\right) \longmapsto(u-\eta \Phi)(s)-(v+\eta \Phi)\left(s^{\prime}\right)-\psi_{n}\left(s, s^{\prime}\right) .
$$

Then applying Theorem 3.2 in [9] to the upper-semicontinuous functions $u-\eta \Phi$ and to the lower-semicontinuous function $v+\eta \Phi$, we deduce that there exist $3 \times 3$ symmetric matrices $\Xi_{n}$ and $\Upsilon_{n}$, with $\Xi_{n} \leq \Upsilon_{n}$ such that

$$
\begin{aligned}
j_{n} & :=\left(q_{n}:=D_{1} \psi\left(s_{n}, s_{n}^{\prime}\right)+\eta D \Phi\left(s_{n}\right) ; Q_{n}:=\Xi_{n}+\eta D^{2} \Phi\left(s_{n}\right)\right) \in \bar{J}_{\overline{\mathcal{S}} \backslash \partial^{z} \mathcal{S}}^{2,+} u\left(s_{n}\right)(4.9) \\
j_{n}^{\prime} & :=\left(q_{n}^{\prime}:=-D_{2} \psi\left(s_{n}, s_{n}^{\prime}\right)-\eta D \Phi\left(s_{n}^{\prime}\right) ; Q_{n}^{\prime}:=\Upsilon_{n}-\eta D^{2} \Phi\left(s_{n}^{\prime}\right)\right) \in \bar{J}_{\mathcal{S}}^{2,-} v\left(s_{n}^{\prime} \backslash 4,10\right)
\end{aligned}
$$

and

$$
-\left(2 n^{2}+\left\|M_{n}\right\|\right) I \leq\left(\begin{array}{cc}
\Xi_{n} & 0 \\
0 & -\Upsilon_{n}
\end{array}\right) \leq M_{n}+\frac{1}{2 n^{2}} M-n^{2}
$$

where

$$
M_{n}:=D^{2} \psi\left(s_{n}, s_{n}^{\prime}\right)=2 n^{2}\left(\begin{array}{cc}
I & -I \\
-I & I
\end{array}\right)+2 \zeta\left(\begin{array}{ll}
I & 0 \\
0 & 0
\end{array}\right)
$$

$D_{1} \psi\left(s, s^{\prime}\right)=2 n\left(n\left(s-s^{\prime}\right)-\zeta \mathbf{g}^{\mathbf{b}}\right)+2 \zeta\left(s-s_{0}\right), \quad-D_{2} \psi\left(s, s^{\prime}\right)=2 n\left(n\left(s-s^{\prime}\right)-\zeta \mathbf{g}^{\mathbf{b}}\right)$.

Here the norm of a symmetric matrix $M$ is defined as $\|M\|=\sup \{M \xi \cdot \xi:|\xi| \leq 1\}$.

By (4.8) and for large $n \geq 1$, the subsolution property of $u$ holds at $j_{n}$ and the supersolution property of $v$ holds at $j_{n}^{\prime}$, i.e.

$$
\begin{aligned}
& \min \left\{\beta u\left(s_{n}\right)-\tilde{\mathcal{L}}\left(s_{n}, q_{n}, Q_{n}\right)-\tilde{U}\left(q_{n 1}\right), \mathbf{g}^{\mathbf{b}} \cdot q_{n}, \mathbf{g}_{\varepsilon}^{\mathbf{s}}\left(s_{n}\right) \cdot q_{n}\right\} \leq 0, \\
& \min \left\{\beta v\left(s_{n}^{\prime}\right)-\tilde{\mathcal{L}}\left(s_{n}^{\prime}, q_{n}^{\prime}, Q_{n}^{\prime}\right)-\tilde{U}\left(q_{n 1}^{\prime}\right), \mathbf{g}^{\mathbf{b}} \cdot q_{n}^{\prime}, \mathbf{g}_{\varepsilon}^{\mathbf{s}}\left(s_{n}^{\prime}\right) \cdot q_{n}^{\prime}\right\} \geq 0 .
\end{aligned}
$$

3. For each $n \geq 1$

$$
\mathbf{g}^{\mathbf{b}} \cdot q_{n}-\mathbf{g}^{\mathbf{b}} \cdot q_{n}^{\prime}=\eta \mathbf{g}^{\mathbf{b}} \cdot\left(D \Phi\left(s_{n}\right)+D \Phi\left(s_{n}^{\prime}\right)\right)+2 \zeta \mathbf{g}^{\mathbf{b}} \cdot\left(s_{n}-s_{0}\right) .
$$

Recall that $s_{n}, s_{n}^{\prime} \longrightarrow s_{0} \in \overline{\mathcal{S}} \backslash \partial^{z} \mathcal{S}$, and $\mathbf{g}^{\mathbf{b}} \cdot \Phi>0$ on $\overline{\mathcal{S}} \backslash \partial^{z} \mathcal{S}$, then

$$
\lim _{n \rightarrow \infty}\left(\mathbf{g}^{\mathbf{b}} \cdot q_{n}-\mathbf{g}^{\mathbf{b}} \cdot q_{n}^{\prime}\right)=2 \eta \mathbf{g}^{\mathbf{b}} \cdot D \Phi\left(s_{0}\right)>0 .
$$

We also compute for all $n \geq 1$ that

$$
\begin{gathered}
\mathbf{g}_{\varepsilon}^{\mathbf{s}}\left(s_{n}\right) \cdot q_{n}-\mathbf{g}_{\varepsilon}^{\mathbf{s}}\left(s_{n}^{\prime}\right) \cdot q_{n}^{\prime}=\eta\left(\mathbf{g}_{\varepsilon}^{\mathbf{s}}\left(s_{n}\right) \cdot D \Phi\left(s_{n}\right)+\mathbf{g}_{\varepsilon}^{\mathbf{s}}\left(s_{n}^{\prime}\right) \cdot D \Phi\left(s_{n}^{\prime}\right)\right)+2 \zeta \mathbf{g}_{\varepsilon}^{\mathbf{s}}\left(s_{n}\right) \cdot\left(s_{n}-s_{0}\right) \\
+\left(\mathbf{g}_{\varepsilon}^{\mathbf{s}}\left(s_{n}\right)-\mathbf{g}_{\varepsilon}^{\mathbf{s}}\left(s_{n}^{\prime}\right)\right) \cdot 2 n\left[n\left(s_{n}-s_{n}^{\prime}\right)-\zeta \mathbf{g}^{\mathbf{b}}\right] .
\end{gathered}
$$


By the local Lipschitz continuity of the function $\mathbf{g}_{\varepsilon}^{\mathbf{s}}$ at $s_{0}$, there exists some positive constant $C_{0}$ such that for large $n$

$$
\begin{aligned}
& \left|\mathbf{g}_{\varepsilon}^{\mathbf{s}}\left(s_{n}\right) \cdot q_{n}-\mathbf{g}_{\varepsilon}^{\mathbf{s}}\left(s_{n}^{\prime}\right) \cdot q_{n}^{\prime}-\eta\left(\mathbf{g}_{\varepsilon}^{\mathbf{s}}\left(s_{n}\right) \cdot D \Phi\left(s_{n}\right)+\mathbf{g}_{\varepsilon}^{\mathbf{s}}\left(s_{n}^{\prime}\right) \cdot D \Phi\left(s_{n}^{\prime}\right)\right)\right| \\
& \quad \leq 2 \zeta\left|\mathbf{g}_{\varepsilon}^{\mathbf{s}}\left(s_{n}\right)\right|\left|s_{n}-s_{0}\right| C_{0}\left|s_{n}-s_{n}^{\prime}\right| 2 n\left|n\left(s_{n}-s_{n}^{\prime}\right)-\zeta \mathbf{g}^{\mathbf{b}}\right| \\
& \quad \leq 2 \zeta\left|\mathbf{g}_{\varepsilon}^{\mathbf{s}}\left(s_{n}\right)\right|\left|s_{n}-s_{0}\right| 2 C_{0}\left|n\left(s_{n}-s_{n}^{\prime}\right)-\zeta \mathbf{g}^{\mathbf{b}}\right|^{2}+2 C_{0} \zeta\left|\mathbf{g}^{\mathbf{b}}\right|\left|n\left(s_{n}-s_{n}^{\prime}\right)-\zeta \mathbf{g}^{\mathbf{b}}\right| .
\end{aligned}
$$

Since $s_{n} \longrightarrow s_{0}$ and $\left|n\left(s_{n}-s_{n}^{\prime}\right)-\zeta \mathbf{g}^{\mathbf{b}}\right| \longrightarrow 0$, we get

$$
\lim _{n \rightarrow \infty}\left(\mathbf{g}_{\varepsilon}^{\mathbf{s}}\left(s_{n}\right) \cdot q_{n}-\mathbf{g}_{\varepsilon}^{\mathbf{s}}\left(s_{n}^{\prime}\right) \cdot q_{n}^{\prime}\right)=2 \eta \mathbf{g}_{\varepsilon}^{\mathbf{s}} \cdot D \Phi\left(s_{0}\right)>0 .
$$

We deduce from (4.13), (4.14), (4.15) together with Lemma 4.2 that for large $n$,

$$
\min \left\{\mathbf{g}^{\mathbf{b}} \cdot q_{n}, \mathbf{g}_{\varepsilon}^{\mathbf{s}}\left(s_{n}\right) \cdot q_{n}\right\} \geq 2 \min \left\{\mathbf{g}^{\mathbf{b}} \cdot D \Phi\left(s_{0}\right), \mathbf{g}_{\varepsilon}^{\mathbf{s}}\left(s_{n}\right) \cdot D \Phi\left(s_{0}\right)\right]+o(1)>0 .
$$

Consequently, (4.12) implies that for large $n$

$$
\beta u\left(s_{n}\right)-\tilde{\mathcal{L}}\left(s_{n}, q_{n}, Q_{n}\right)-\tilde{U}\left(q_{n 1}\right) \leq 0 .
$$

4. From (4.13) and (4.16), it follows that for large $n$

$$
\beta u\left(s_{n}\right)-\tilde{\mathcal{L}}\left(s_{n}, q_{n}, Q_{n}\right)-\tilde{U}\left(q_{n 1}\right) \leq 0 \leq \beta v\left(s_{n}^{\prime}\right)-\tilde{\mathcal{L}}\left(s_{n}^{\prime}, q_{n}^{\prime}, Q_{n}^{\prime}\right)-\tilde{U}\left(q_{n 1}^{\prime}\right) .
$$

Using the local Lipschitz continuity property of the function $\tilde{U}$, a direct calculation shows that for some positive constant $C$ and for large $n$,

$$
\begin{aligned}
& \beta\left(u\left(s_{n}\right)-v\left(s_{n}^{\prime}\right)\right) \leq \tilde{\mathcal{L}}\left(s_{n}, q_{n}, Q_{n}\right)-\tilde{\mathcal{L}}\left(s_{n}^{\prime}, q_{n}^{\prime}, Q_{n}^{\prime}\right)+\tilde{U}\left(q_{n 1}\right)-\tilde{U}\left(q_{n 1}^{\prime}\right) \\
& \leq C\left(\left|s_{n}\right| \zeta\left|s_{n}-s_{0}\right|+\left|n\left(s_{n}-s_{n}^{\prime}\right)-\zeta \mathbf{g}^{\mathbf{b}}\right|^{2}+\left|D \Phi\left(s_{n}\right)-D \Phi\left(s_{n}^{\prime}\right)\right|\right) \\
&+\frac{\sigma^{2}}{2}\left(y_{n}^{2}\left(Q_{n}\right)_{22}-\left(y_{n}^{\prime}\right)^{2}\left(Q_{n}^{\prime}\right)_{22}\right) \\
&+\eta\left\{\tilde{\mathcal{L}}\left(s_{n}, D \Phi\left(s_{n}\right), D^{2} \Phi\left(s_{n}\right)\right)+\tilde{\mathcal{L}}\left(s_{n}^{\prime}, D \Phi\left(s_{n}^{\prime}\right), D^{2} \Phi\left(s_{n}^{\prime}\right)\right)\right\} .
\end{aligned}
$$

From (4.11), we have that

$$
\left(y_{n}^{2}\left(Q_{n}\right)_{22}-\left(y_{n}^{\prime}\right)^{2}\left(Q_{n}^{\prime}\right)_{22}\right) \leq 4 \zeta y_{n}\left(u_{n}-y_{n}^{\prime}\right)+\frac{\zeta^{2}}{n^{2}} y_{n} .
$$

Moreover, the mapping $\Phi$ satisfies $\beta \Phi()-.\tilde{\mathcal{L}}\left(., D \Phi, D^{2} \Phi\right)$ on $\overline{\mathcal{S}} \backslash \partial^{z} \mathcal{S}$, hence for some positive constant $\tilde{C}$ and for large $n$

$$
\begin{aligned}
\beta\left[u\left(s_{n}\right)-v\left(s_{n}^{\prime}\right)\right]-\eta \Phi\left(s_{n}\right)-\eta \Phi\left(s_{n}^{\prime}\right) \leq \tilde{\mathcal{L}}\left(s_{n}, q_{n}, Q_{n}\right)-\tilde{\mathcal{L}}\left(s_{n}^{\prime}, q_{n}^{\prime}, Q_{n}^{\prime}\right)+\tilde{U}\left(q_{n 1}\right)-\tilde{U}\left(q_{n 1}^{\prime}\right) \\
\leq \tilde{C}\left\{\frac{1}{n^{2}}+\left|s_{n}\right| \zeta\left|s_{n}-s_{0}\right|+\left|n\left(s_{n}-s_{n}^{\prime}\right)-\zeta \mathbf{g}^{\mathbf{b}}\right|^{2}\right. \\
\left.+\left|D \Phi\left(s_{n}\right)-D \Phi\left(s_{n}^{\prime}\right)\right|\right\}
\end{aligned}
$$

where the right-hand side of the inequality goes to zero as $n \rightarrow \infty$. This implies

$$
\beta\left[u\left(s_{0}\right)-v\left(s_{0}\right)\right]-2 \eta \Phi\left(s_{0}\right)=\limsup _{n \rightarrow \infty}\left(\beta\left[u\left(s_{n}\right)-v\left(s_{n}^{\prime}\right)\right]-\eta \Phi\left(s_{n}\right)-\eta \Phi\left(s_{n}^{\prime}\right)\right) \leq 0,
$$

contradicting (4.5). 
We conclude this section by the

Proof of Proposition 2.3 We use the same arguments as in the proof of Theorem 3.1 , substituting $\mathbf{g}^{\mathbf{s}}$ to $\mathbf{g}_{\varepsilon}^{\mathbf{s}}$. The only difference is the following. The maximizer $s_{0}$ in (4.5) is now known to be in $\overline{\mathcal{S}} \backslash\left(\partial^{z} \mathcal{S} \cup\{(x, 0,0): x \geq 0\}\right)$, as it is assumed in the statement of the proposition that $u \leq v$ on $\partial^{z} \mathcal{S} \cup\{(x, 0,0): x \geq 0\}$. Then, the sequences $\left(s_{n}\right)_{n}$ and $\left(s_{n}^{\prime}\right)_{n}$, defined in Step 1, are valued in a ball around $s_{0}$ which does not intersect the axis $\{(x, 0,0): x \geq 0\}$. Since $\mathbf{g}^{\mathbf{s}}$ is locally Lipschitz on $\overline{\mathcal{S}} \backslash\{(x, 0,0): x \geq 0\}$, we just follow the lines of the previous proof.

5. An approximating control problem. Let $s=(x, y, k)$ be an initial condition in the state space $\overline{\mathcal{S}}$, and consider a control process $\nu \in \mathcal{A}$, i.e. a triple of $\mathbb{F}$-adapted processes $\nu=(C, L, M)$, with non-decreasing right-continuous processes $L, M, L_{0-}=M_{0-}=0$, and satisfying Conditions (2.2) and (2.3). For every parameter $\varepsilon \geq 0$, we denote by $S^{\varepsilon, s, \nu}=\left(X^{\varepsilon, s, \nu}, Y^{\varepsilon, s, \nu}, K^{\varepsilon, s, \nu}\right)$ the unique strong solution of

$$
\begin{aligned}
& d X_{t}^{\varepsilon}=\left(r X_{t}^{\varepsilon}-C_{t}\right) d t-(1+\lambda) d L_{t}+(1-\mu)\left[(1-\alpha) Y_{t-}^{\varepsilon}+\alpha f^{\varepsilon}\left(S_{t-}^{\varepsilon}\right) K_{t-}^{\varepsilon}\right] d M_{t}(5.1) \\
& d Y_{t}^{\varepsilon}=Y_{t}^{\varepsilon}\left[\rho d t+\sigma d W_{t}\right]+d L_{t}-Y_{t-}^{\varepsilon} d M_{t} \\
& d K_{t}^{\varepsilon}=d L_{t}-f^{\varepsilon}\left(S_{t-}^{\varepsilon}\right) K_{t-}^{\varepsilon} d M_{t}
\end{aligned}
$$

with initial condition $S_{0-}^{\varepsilon, s, \nu}=s$. With this definition, observe that the jumps of the state processes $S^{\varepsilon, s, \nu}$ are given by

$$
\Delta S_{t}^{\varepsilon, s, \nu}=-\Delta L_{t} \mathbf{g}^{\mathbf{b}}-\Delta M_{t}\left[(1-\alpha) Y_{t-}^{\varepsilon, s, \nu}+\alpha f^{\varepsilon}\left(S_{t-}^{\varepsilon, s, \nu}\right) K_{t-}^{\varepsilon, s, \nu}\right] \mathbf{g}_{\varepsilon}^{\mathbf{s}}\left(S_{t-}^{\varepsilon, s, \nu}\right)
$$

where the vector fields $\mathbf{g}^{\mathbf{b}}$ and $\mathbf{g}_{\varepsilon}^{\mathbf{s}}$ are defined in (2.15) and (3.2).

A control process $\nu=(C, L, M)$ is said to be $(s, \varepsilon)$-admissible if the corresponding state process $S^{\varepsilon, s, \nu}$ is valued in $\overline{\mathcal{S}}$. We shall denote by $\mathcal{A}^{\varepsilon}(s)$ the collection of all $(s, \varepsilon)$-admissible controls.

For every initial condition $s \in \overline{\mathcal{S}}, \varepsilon \geq 0$, and $(\varepsilon, s)$-admissible control $\nu=$ $(C, L, M)$, we introduce the criterion

$$
J_{T}^{\varepsilon}(s, \nu):=\mathbb{E}\left[\int_{0}^{T} e^{-\beta t} U\left(C_{t}\right) d t+e^{-\beta T} U\left(Z_{T}^{\varepsilon, s, \nu}\right) \mathbf{1}_{T<\infty}\right], T \in \mathbb{R}_{+} \cup\{\infty\},
$$

where $U$ is the power utility function defined in (2.8). The value function $V_{\varepsilon}$ is then defined by

$$
V_{\varepsilon}(s):=\sup _{\nu \in \mathcal{A}^{\varepsilon}(s)} J_{\infty}^{\varepsilon}(s, \nu)
$$

REMARK 2. When $\varepsilon=0$, the above problem reduces to the optimal investment problem under capital gains taxes reviewed in Section 2, in particular $V_{0}=V$. For positive $\varepsilon$, the control problem (5.5) can be interpreted as a utility maximization problem with a modified taxation rule. Under this new taxation rule, the tax basis used to evaluate the capital gains is equal to the relative weighted average purchase price as long as the ratio $K / Z$ is larger then $2 \varepsilon$, but it is set to zero when $K / Z<\varepsilon$. Roughly speaking, for $\varepsilon>0$, the investor pays more taxes then in the original market when the ratio $K / Z<\varepsilon$. Consequently, we expect that $V_{\varepsilon}$ increases towards $V$ as $\varepsilon$ goes to zero. This will be proved in Proposition 6.2 below. 
The main objective of this paragraph is to prove that the function $V_{\varepsilon}$ is a constrained viscosity solution of the approximating PDE (3.3), thus proving the existence statement in Theorem 3.2. The arguments of this section hold for every $\varepsilon \geq 0$. In particular, the proof of Proposition 2.2 corresponds to the special case $\varepsilon=0$.

As usual, the key-ingredient in order to derive the dynamic programming equation is a dynamic programming principle. We state it here without proof, and we refer to $[6],[13]$ and [14].

TheOREM 5.1. Let $\varepsilon \geq 0, s \in \overline{\mathcal{S}}$, and let $\tau$ be some $\mathbb{P}-$ a.s. finite $\mathbb{F}$-stopping time. Then

$$
V_{\varepsilon}(s)=\sup _{\nu=(C, L, M) \in \mathcal{A}^{\varepsilon}(s)} \mathbb{E}\left[\int_{0}^{\tau} e^{-\beta t} U\left(C_{t}\right) d t+e^{-\beta \tau} V_{\varepsilon}\left(S_{\tau}^{\varepsilon, s, \nu}\right)\right] .
$$

Before turning to the derivation of the dynamic programming equation for the problem $V_{\varepsilon}$, we introduce a notation which will be used frequently in the sequel. Let $\varepsilon \geq 0, s \in \mathcal{\mathcal { S }}, \nu=(C, L, M) \in \mathcal{A}(s)$, and consider some stopping time $\tau$ such that $S_{\tau-}^{\overline{\varepsilon, s}, \nu} \in \overline{\mathcal{S}}$. Then, it is easy to verify that the strategy $\nu(\tau)$ defined by

$$
\nu(\tau)_{t}:=(\bar{C}, \bar{L}, \bar{M}):=\nu_{t} \mathbf{1}_{[0, \tau[}(t)+\left(0, L_{\tau-}, M_{\tau-}+\left(1-\Delta M_{\tau}\right)\right) \mathbf{1}_{[\tau, \infty)}(t)
$$

is in $\mathcal{A}^{\varepsilon}(s)$, and that

$$
\mathbb{E}\left[\int_{0}^{\infty} e^{-\beta t} U\left(\bar{C}_{t}\right) d t\right]=\mathbb{E}\left[\int_{0}^{\tau} e^{-\beta t} U\left(\bar{C}_{t}\right) d t\right]
$$

5.1. Supersolution property. In this section, we prove that the value function $V_{\varepsilon}$ is a viscosity supersolution of $(3.3)$ on $\mathcal{S}$ for every $\varepsilon \geq 0$.

1. Fix some $\varepsilon \geq 0$. Recall that $V_{\varepsilon} \geq 0$ by definition, and in particular $\left(V_{\varepsilon}\right)_{*}(0) \geq 0$. So it remains to show that, for $s_{0}$ be in $\mathcal{S}$ and $\varphi$ in $C^{2}(\overline{\mathcal{S}})$ such that

$$
0=\left(\left(V_{\varepsilon}\right)_{*}-\varphi\right)\left(s_{0}\right)=\min _{\mathcal{S}}\left(\left(V_{\varepsilon}\right)_{*}-\varphi\right),
$$

the test function $\varphi$ must satisfy at the point $s_{0}$ :

$$
\min \left\{-\mathcal{L} \varphi, \mathbf{g}^{\mathbf{b}} \cdot D \varphi, \mathbf{g}_{\varepsilon}^{\mathbf{s}} \cdot D \varphi\right\}\left(s_{0}\right) \geq 0 .
$$

2.1. Let $\eta>0$ be such that $B\left(s_{0}, \eta\right) \subset \mathcal{S}$, and consider some sequence $\left(s_{n}\right)_{n \geq 1}$ satisfying

$$
\begin{aligned}
& \text { (i) } B\left(s_{0}, \eta\right) \ni s_{n} \underset{n \rightarrow \infty}{\longrightarrow} s_{0}, \\
& \text { (ii) } \xi_{n}:=V_{\varepsilon}\left(s_{n}\right)-\varphi\left(s_{n}\right) \longrightarrow 0 \text { as } n \rightarrow 0 .
\end{aligned}
$$

Fix some $(c, \ell, m)$ in $(0, \infty)^{3}$, define the strategy $\nu \in \mathcal{A}$ by $: \nu_{t}=\left(C_{t}=c, L_{t}=\ell t, M_{t}=m t\right)$, and let $\left(\tau^{n}\right)_{n \geq 0}$ be the stopping times

$$
\tau^{n}:=\inf \left\{t \geq 0: S_{t}^{\varepsilon, s_{n}, \nu} \notin \mathcal{S}\right\} n \geq 0 .
$$

Given that for each $n \geq 0, s_{n} \notin \partial^{z} \mathcal{S}$, and that the strategy $\nu$ is continuous, we have

$$
\tau^{n}>0 \text { for all } n \geq 0 \text { and } \tau^{n} \underset{n \rightarrow \infty}{\longrightarrow} \tau^{0} \mathbb{P}-\text { a.s. }
$$

2.2. To each $n \geq 1$ we associate the $\left(\varepsilon, s_{n}\right)$-admissible strategy $\nu\left(\tau^{n}\right)=\left(C^{n}, L^{n}, M^{n}\right)$ $\in \mathcal{A}^{\varepsilon}\left(s_{n}\right)$ defined in (5.6). To simplify the notations, we set $S^{n}:=S^{\varepsilon, s_{n}, \nu\left(\varepsilon, s_{n}\right)}$. For 
any $\mathbb{P}$ - a.s. finite stopping time $\theta^{n}$, the dynamic programming principle of Theorem 5.1 provides

$$
V_{\varepsilon}\left(s_{n}\right) \geq \mathbb{E}\left[\int_{0}^{\theta^{n} \wedge \tau^{n} / 2} e^{-\beta t} U\left(C_{t}^{n}\right) d t+e^{-\beta \theta^{n} \wedge \tau^{n} / 2} V_{\varepsilon}\left(S_{\theta^{n} \wedge \tau^{n} / 2}^{n}\right)\right]
$$

Notice that $S_{\theta^{n} \wedge \tau^{n} / 2}^{n} \in \mathcal{S}$, we then deduce from the inequalities $\varphi \leq\left(V_{\varepsilon}\right)_{*} \leq V_{\varepsilon}$ on $\mathcal{S}$ that

$$
\xi_{n}+\varphi\left(s_{n}\right) \geq \mathbb{E}\left[\int_{0}^{\theta^{n} \wedge \tau^{n} / 2} e^{-\beta t} U\left(C_{t}^{n}\right) d t+e^{-\beta \theta^{n} \wedge \tau^{n} / 2} \varphi\left(S_{\theta^{n} \wedge \tau^{n} / 2}^{n}\right)\right]
$$

By the definition of the strategy $\nu\left(\tau^{n}\right)$, jumps of the process $S^{n}$ may occur only at the stopping time $\tau^{n}$, and by definition of the stopping time $\tau^{n}$, the process $\left\{S_{t}^{n} \mathbf{1}_{\left[0, \tau^{n}\right]}(t), t \geq 0\right\}$ is uniformly bounded. Hence, using Itô formula we get

$$
\begin{aligned}
-\xi_{n} \leq \mathbb{E} & {\left[\int_{0}^{\theta^{n} \wedge \tau^{n} / 2} e^{-\beta t}\left\{-\mathcal{L} \varphi+\tilde{U}\left(\varphi_{x}\right)-\left(U\left(C_{t}^{n}\right)-C_{t}^{n} \varphi_{x}\right)\right\}\left(S_{t}^{n}\right) d t\right] } \\
+ & \ell \mathbb{E}\left[\int_{0}^{\theta^{n} \wedge \tau^{n} / 2} e^{-\beta t} \mathbf{g}^{\mathbf{b}} \cdot D \varphi\left(S_{t}^{n}\right) d t\right] \\
& +m \mathbb{E}\left[\int_{0}^{\theta^{n} \wedge \tau^{n} / 2} e^{-\beta t}\left[(1-\alpha) Y_{t}^{n}+\alpha f^{\varepsilon}\left(S_{t}^{n}\right) K_{t}^{n}\right]\left(\mathbf{g}_{\varepsilon}^{\mathbf{s}} \cdot D \varphi\right)\left(S_{t}^{n}\right) d t\right] .
\end{aligned}
$$

2.3. Set

$$
\theta_{n}= \begin{cases}\sqrt{\xi_{n}} & \text { if } \xi_{n}>0 \\ n^{-1} & \text { if } \xi_{n}=0\end{cases}
$$

Since $\theta^{n} \longrightarrow 0$ and $\tau^{n} \longrightarrow \tau^{0}>0 \mathbb{P}-$ a.s. as $n \rightarrow \infty$, it follows that for $\mathbb{P}-$ a.e $\omega$, $\theta^{n} \wedge \tau^{n} / 2=\theta^{n}$ for large $n$. Rewriting (5.9), and taking the limits as $n \rightarrow \infty$, we obtain

$$
\begin{aligned}
0=\lim _{n \rightarrow \infty}-\frac{\xi_{n}}{\theta_{n}} & \\
\leq \liminf _{n \rightarrow \infty} \mathbb{E} & {\left[\frac{1}{\theta_{n}} \int_{0}^{\theta^{n} \wedge \tau^{n} / 2} e^{-\beta t}\left\{-\mathcal{L} \varphi+\tilde{U}\left(\varphi_{x}\right)-\left(U\left(C_{t}^{n}\right)-C_{t}^{n} \varphi_{x}\right)\right\}\left(S_{t}^{n}\right) d t\right] } \\
+ & \ell \mathbb{E}\left[\frac{1}{\theta_{n}} \int_{0}^{\theta^{n} \wedge \tau^{n} / 2} e^{-\beta t} \mathbf{g}^{\mathbf{b}} \cdot D \varphi\left(S_{t}^{n}\right) d t\right] \\
& +m \mathbb{E}\left[\frac{1}{\theta_{n}} \int_{0}^{\theta^{n} \wedge \tau^{n} / 2} e^{-\beta t}\left[(1-\alpha) Y_{t}^{n}+\alpha f^{\varepsilon}\left(S_{t}^{n}\right) K_{t}^{n}\right]\left(\mathbf{g}_{\varepsilon}^{\mathbf{s}} \cdot D \varphi\right)\left(S_{t}^{n}\right) d t\right] .
\end{aligned}
$$

Since $\varphi \in C^{2}(\overline{\mathcal{S}})$ and the process $\left\{S_{t}^{n} \mathbf{1}_{\left[0, \tau^{n} / 2\right]}(t), t \geq 0\right\}$ is continuous and uniformly 
bounded, we get by dominated convergence

$$
\begin{aligned}
& \liminf _{n \rightarrow \infty} \mathbb{E} {\left[\frac{1}{\theta_{n}} \int_{0}^{\theta^{n} \wedge \tau^{n} / 2} e^{-\beta t}\left\{-\mathcal{L} \varphi+\tilde{U}\left(\varphi_{x}\right)-\left(U\left(C_{t}^{n}\right)-C_{t}^{n} \varphi_{x}\right)\right\}\left(S_{t}^{n}\right) d t\right] } \\
&+ \ell \mathbb{E}\left[\frac{1}{\theta_{n}} \int_{0}^{\theta^{n} \wedge \tau^{n} / 2} e^{-\beta t} \mathbf{g}^{\mathbf{b}} \cdot D \varphi\left(S_{t}^{n}\right) d t\right] \\
&+m \mathbb{E}\left[\frac{1}{\theta_{n}} \int_{0}^{\theta^{n} \wedge \tau^{n} / 2} e^{-\beta t}\left[(1-\alpha) Y_{t}^{n}+\alpha f^{\varepsilon}\left(S_{t}^{n}\right) K_{t}^{n}\right]\left(\mathbf{g}_{\varepsilon}^{\mathbf{s}} \cdot D \varphi\right)\left(S_{t}^{n}\right) d t\right] . \\
&=-\mathcal{L} \varphi\left(s_{0}\right)+\tilde{U}\left(\varphi_{x}\left(s_{0}\right)-\left(U(c)-c \varphi_{x}\left(s_{0}\right)\right)\right. \\
&+\ell \mathbf{g}^{\mathbf{b}} \cdot D \varphi\left(s_{0}\right)+m\left[(1-\alpha) y_{0}+\alpha f^{\varepsilon}\left(s_{0}\right) k_{0}\right] \mathbf{g}_{\varepsilon}^{\mathbf{s}}\left(s_{0}\right) \cdot D \varphi\left(s_{0}\right) .
\end{aligned}
$$

Recall (5.10), then

$$
\begin{aligned}
0 \leq-\mathcal{L} \varphi\left(s_{0}\right)+\tilde{U}\left(\varphi_{x}\left(s_{0}\right)-\left(U(c)-c \varphi_{x}\left(s_{0}\right)\right)\right. \\
+\ell \mathbf{g}^{\mathbf{b}} \cdot D \varphi\left(s_{0}\right)+m\left[(1-\alpha) y_{0}+\alpha f^{\varepsilon}\left(s_{0}\right) k_{0}\right] \mathbf{g}_{\varepsilon}^{\mathbf{s}}\left(s_{0}\right) \cdot D \varphi\left(s_{0}\right) .
\end{aligned}
$$

2.4. Observe that $s_{0} \in \mathcal{S}$ implies that $\left[(1-\alpha) y_{0}+\alpha f^{\mathcal{E}}\left(s_{0}\right) k_{0}\right]>0$. Since $(c, \ell, m) \in$ $(0, \infty)^{3},(5.11)$ provides

$$
0 \leq \min \left\{-\mathcal{L} \varphi, \mathbf{g}^{\mathbf{b}} \cdot D \varphi, \mathbf{g}_{\varepsilon}^{\mathbf{s}} \cdot D \varphi\right\}\left(s_{0}\right) .
$$

5.2. Subsolution property. In this section, we prove that the value function $V_{\varepsilon}$ is a constrained viscosity subsolution of (3.3) for every $\varepsilon \geq 0$. In preparation for this proof, we state some intermediate results.

Lemma 5.2. Let $\varphi$ be a mapping in $C^{2}(\overline{\mathcal{S}})$, and $s_{0} \in \overline{\mathcal{S}}$ such that $\varphi_{x}\left(s_{0}\right)>0$. Then there exists $\eta>0, \gamma>0$, and $c_{0}>0$ such that

$$
\tilde{U}\left(\varphi_{x}(s)\right)-\left[U(c)-c \varphi_{x}(s)\right] \geq \gamma\left(c-c_{0}\right)^{+} \text {for allc } \geq 0 \text { and } s \in B\left(s_{0}, \eta\right) \cap \overline{\mathcal{S}} .
$$

Proof. Since $\varphi_{x}\left(s_{0}\right)>0$ we can find some $\eta, \delta>0$ such that $\varphi_{x}>\delta$ on $B\left(s_{0}, \eta\right) \cap \overline{\mathcal{S}}$. The mapping $s \longmapsto \mathcal{I}\left(\varphi_{x}(s)\right):=\left(U^{\prime}\right)^{-1}\left(\varphi_{x}(s)\right)$ is then bounded on $B\left(s_{0}, \eta\right) \cap \overline{\mathcal{S}}$, and since $U^{\prime}$ is a decreasing function, we can find $c_{0}>0$ such that

$$
c_{0}>\max _{B\left(s_{0}, \eta\right) \cap \overline{\mathcal{S}}} \mathcal{I}\left(\varphi_{x}\right) \text { and } \gamma:=\min _{B\left(s_{0}, \eta\right) \cap \overline{\mathcal{S}}}\left(\varphi_{x}-U^{\prime}\left(c_{0}\right)\right)>0 .
$$

For all $s \in B\left(s_{0}, \eta\right) \cap \overline{\mathcal{S}}$, using the nonnegativity and the convexity of the function $c \in \mathbb{R}_{+} \longmapsto \tilde{U}\left(\varphi_{x}(s)\right)-\left(U(c)-c \varphi_{x}(s)\right)$, we get :

$$
\begin{aligned}
\tilde{U}\left(\varphi_{x}(s)\right)-\left(U(c)-c \varphi_{x}(s)\right) \geq \tilde{U}\left(\varphi_{x}(s)\right)-\left(U(c)-c \varphi_{x}(s)\right) & -\tilde{U}\left(\varphi_{x}(s)\right)+\left(U\left(c_{0}\right)-c_{0} \varphi_{x}(s)\right) \\
& \geq\left(\varphi_{x}(s)-U^{\prime}\left(c_{0}\right)\right)\left(c-c_{0}\right)^{+} \\
\geq & \gamma\left(c-c_{0}\right)^{+} .
\end{aligned}
$$

Lemma 5.3. Let $\varphi \in \mathrm{C}^{1}(\overline{\mathcal{S}})$ and $s_{0} \in \overline{\mathcal{S}} \backslash \partial^{z} \mathcal{S}$. Assume that $\min \left\{\mathbf{g}^{\mathbf{b}} \cdot D \varphi, \mathbf{g}_{\varepsilon}^{\mathbf{s}} \cdot D \varphi\right\}\left(s_{0}\right)>$ 0 . Then, there exist $\eta, \gamma>0$ such that for $s=(x, y, k) \in B\left(s_{0}, \eta\right) \cap \overline{\mathcal{S}}$, and $s^{\prime}:=s-\ell \mathbf{g}^{\mathbf{b}} \eta-m\left[(1-\alpha) y+\alpha f^{\varepsilon}(s) k\right] \mathbf{g}_{\varepsilon}^{\mathbf{s}} \in B\left(s_{0}, \eta\right) \cap \overline{\mathcal{S}}$ with $\ell, m \geq 0$,

$$
\varphi(s)-\varphi\left(s^{\prime}\right) \geq \gamma \ell+\gamma m\left[(1-\alpha) y+\alpha f^{\varepsilon}(s) k\right] .
$$


Proof. We first observe that $\left\|\mathbf{g}_{\varepsilon}^{\mathbf{s}}\right\|_{\infty}<\infty$. In view of the definition of $\mathbf{g}_{\varepsilon}^{\mathbf{s}}$ this follows from that

$$
0 \leq \frac{k f^{\varepsilon}(s)}{(1-\alpha) y+\alpha k f^{\varepsilon}(s)} \leq \frac{k}{(1-\alpha) y+\alpha k} \leq \frac{1}{\alpha},
$$

where we used the inequality $f^{\varepsilon} \leq 1$. Set

$$
4 \gamma:=\min \left\{\mathbf{g}^{\mathbf{b}} \cdot D \varphi ; \mathbf{g}_{\varepsilon}^{\mathbf{s}} \cdot D \varphi\right\}\left(s_{0}\right)>0 .
$$

Since $\mathbf{g}_{\varepsilon}^{\mathbf{s}}$ and $D \varphi$ are continuous on $\overline{\mathcal{S}} \backslash \partial^{z} \mathcal{S}$, there exists some $\eta>0$ such that for all $s, s^{\prime} \in B\left(s_{0}, \eta\right) \cap \overline{\mathcal{S}}$

$$
\text { (i) } \min \left\{\mathbf{g}^{\mathbf{b}} \cdot D \varphi, \mathbf{g}_{\varepsilon}^{\mathbf{s}} \cdot D \varphi\right\}(s)>2 \gamma \operatorname{and}(\mathrm{ii})\left|D \varphi(s)-D \varphi\left(s^{\prime}\right)\right| \leq \frac{\gamma}{\left\|\mathbf{g}_{\varepsilon}^{\mathbf{s}}\right\|_{\infty}} .
$$

Let $s$ and $s^{\prime}$ be as in the statement of the lemma. By the mean value theorem, there exists some $s^{*} \in\left[s, s^{\prime}\right] \subset B\left(s_{0}, \eta\right) \cap \overline{\mathcal{S}}$ such that

$$
\begin{aligned}
\varphi(s)-\varphi\left(s^{\prime}\right)= & \left(s-s^{\prime}\right) \cdot D \varphi\left(s^{*}\right) \\
= & \ell \mathbf{g}^{\mathbf{b}} \cdot D \varphi\left(s^{*}\right)+m\left[(1-\alpha) y+\alpha f^{\varepsilon}(s) k\right] \mathbf{g}_{\varepsilon}^{\mathbf{s}}(s) \cdot D \varphi\left(s^{*}\right) \\
= & \ell \mathbf{g}^{\mathbf{b}} \cdot D \varphi\left(s^{*}\right)+m\left[(1-\alpha) y+\alpha f^{\varepsilon}(s) k\right] \mathbf{g}_{\varepsilon}^{\mathbf{s}}(s) \cdot D \varphi(s) \\
& \quad-m\left[(1-\alpha) y+\alpha f^{\varepsilon}(s) k\right] \mathbf{g}_{\varepsilon}^{\mathbf{s}}(s) \cdot\left[D \varphi(s)-D \varphi\left(s^{*}\right)\right] \\
\geq & \ell \mathbf{g}^{\mathbf{b}} \cdot D \varphi\left(s^{*}\right)+m\left[(1-\alpha) y+\alpha f^{\varepsilon}(s) k\right] \mathbf{g}_{\varepsilon}^{\mathbf{s}}(s) \cdot D \varphi(s) \\
& \quad-m\left[(1-\alpha) y+\alpha f^{\varepsilon}(s) k\right]\left\|\mathbf{g}_{\varepsilon}^{\mathbf{s}}\right\|_{\infty}\left|D \varphi(s)-D \varphi\left(s^{*}\right)\right| . \\
\geq & \ell 2 \gamma+m\left[(1-\alpha) y+\alpha f^{\varepsilon}(s) k\right](2 \gamma-\gamma) \\
\geq & \gamma \ell+\gamma m\left[(1-\alpha) y+\alpha f^{\varepsilon}(s) k\right] .
\end{aligned}
$$

Proof of the subsolution property 1 . For each $\varepsilon \geq 0$, the value function $V_{\varepsilon}$ is bounded from above by $V$, see Proposition 6.2 below. We also recall from Proposition 4.5 in [5] that for every $s=(x, y, k) \in \overline{\mathcal{S}}$

$$
V(s) \leq V^{0}(x+(1-\mu) \alpha k,(1-\alpha) y),
$$

where the function $V^{0}$, defined in [5], is continuous and satisfies

$$
V^{0}(\bar{x}, \bar{y})=\text { ofor all }(\bar{x}, \bar{y}) \in \mathbb{R}^{2} \text { such that } \bar{x}+(1-\mu) \bar{y}=0 .
$$

It then follows that for each $\varepsilon \geq 0$, the lower-semicontinuous envelope of $V_{\varepsilon}$ satisfies $\left(V_{\varepsilon}\right)_{*} \leq 0$ on $\partial^{z} \mathcal{S}$.

Let $s_{0} \in \overline{\mathcal{S}} \backslash \partial^{z} \mathcal{S}$, and $\varphi \in C^{2}(\overline{\mathcal{S}})$ be such that

$$
0=\left(V_{\varepsilon}^{*}-\varphi\right)\left(s_{0}\right)=\max _{\overline{\mathcal{S}}}\left(V_{\varepsilon}^{*}-\varphi\right),
$$

and assume to the contrary that

$$
F_{*}\left(s_{0}, \varphi\left(s_{0}\right), D \varphi\left(s_{0}\right), D^{2} \varphi\left(s_{0}\right)\right)>0 .
$$


Observe that the last inequality implies that $\tilde{U}\left(\varphi_{x}\left(s_{0}\right)\right)<\infty$ and therefore $\varphi_{x}\left(s_{0}\right)>$ 0 . Since $\varphi \in C^{2}(\overline{\mathcal{S}})$, we deduce from Lemmas 5.2 and 5.3 the existence of $\eta, \gamma, c_{0}>$ 0 , with $B\left(s_{0}, \eta\right) \subset \overline{\mathcal{S}} \backslash \partial^{z} \mathcal{S}$, such that

$$
\begin{gathered}
\min \left\{-\mathcal{L} \varphi, \mathbf{g}^{\mathbf{b}} \cdot D \varphi, \mathbf{g}_{\varepsilon}^{\mathbf{s}} \cdot D \varphi\right\}(s) \wedge \varphi_{x}(s)>0 \\
\tilde{U}\left(\varphi_{x}(s)\right)-\left(U(c)-c \varphi_{x}(s)\right) \geq \gamma\left(c-c_{0}\right) \\
\varphi(s)-\varphi\left(s^{\prime}\right) \geq \gamma l+\gamma m\left[(1-\alpha) y+\alpha f^{\varepsilon}(s) k\right]
\end{gathered}
$$

for all $s \in B\left(s_{0}, \eta\right) \cap \overline{\mathcal{S}}$, and $s^{\prime}=s-\ell \mathbf{g}^{\mathbf{b}} \eta-m \mathbf{g}_{\varepsilon}^{\mathbf{s}} \in B\left(s_{0}, \eta\right) \cap \overline{\mathcal{S}}$ for some $\ell, m \geq 0$.

2. Let $\left(s_{n}=\left(x_{n}, y_{n}, k_{n}\right)\right)_{n>1}$ be some sequence such that

(i) $s_{n} \in B\left(s_{0}, \frac{\eta}{2}\right)$, (ii) $s_{n} \underset{n \rightarrow \infty}{\longrightarrow} s_{0}$, and(iii) $\xi_{n}:=\left|V_{\varepsilon}\left(s_{n}\right)-V_{\varepsilon}^{*}\left(s_{0}\right)\right| \underset{n \rightarrow \infty}{\longrightarrow} 0$.

For each $n \geq 1$, there exists a strategy $\nu^{n}=\left(C^{n}, L^{n}, M^{n}\right) \in \mathcal{A}^{\varepsilon}\left(s_{n}\right)$ such that

$$
V_{\varepsilon}\left(s_{n}\right) \leq \xi_{n}+\mathbb{E}\left[\int_{0}^{\infty} e^{-\beta t} U\left(C_{t}^{n}\right) d t\right]
$$

Set $S^{n}=\left(X^{n}, Y^{n}, K^{n}\right):=S^{\varepsilon, s_{n}, \nu^{n}}$ for $n \geq 1$, and fix some finite positive time horizon $T>0$. By the dynamic programming principle of Theorem 5.1,

$$
V_{\varepsilon}\left(s_{n}\right) \leq \xi_{n}+\mathbb{E}\left[\int_{0}^{T \wedge \theta^{n}} e^{-\beta t} U\left(C_{t}^{n}\right) d t\right]+\mathbb{E}\left[e^{-\beta T \wedge \theta^{n}} V_{\varepsilon}\left(S_{T \wedge \theta^{n}}^{n}\right)\right],
$$

where $\theta^{n}:=\inf \left\{t \geq 0: S_{t}^{n} \notin B\left(s_{0}, \eta\right)\right\}$. Since $V_{\varepsilon} \leq V_{\varepsilon}^{*} \leq \varphi$ on $\overline{\mathcal{S}} \backslash \partial^{z} \mathcal{S}$, and $\xi_{n}=\left|V_{\varepsilon}\left(s_{n}\right)-V_{\varepsilon}^{*}\left(s_{0}\right)\right|=\left|V_{\varepsilon}\left(s_{n}\right)-\varphi\left(s_{0}\right)\right|$, it follows that for all $n \geq 1$ :

$$
\varphi\left(s_{0}\right)-\mathbb{E}\left[e^{-\beta T \wedge \theta^{n}} \varphi\left(S_{T \wedge \theta^{n}}^{n}\right)\right] \leq 2 \xi_{n}+\mathbb{E}\left[\int_{0}^{T \wedge \theta^{n}} e^{-\beta t} U\left(C_{t}^{n}\right) d t\right] .
$$

Notice that for all $n \geq 1$, the process $\left\{S_{t}^{n} \mathbf{1}_{\left[0, T \wedge \theta^{n}\right)}(t), t \geq 0\right\}$ is uniformly bounded, then Itô formula provides

$$
\begin{aligned}
2 \xi_{n} \geq \mathbb{E}\left[\int_{0}^{T \wedge \theta^{n}} e^{-\beta t}\left[-\mathcal{L} \varphi+\tilde{U}\left(\varphi_{x}\right)-\left(U\left(C_{t}^{n}\right)-C_{t}^{n} \varphi_{x}\right)\right]\left(S_{t}^{n}\right) d t\right] \\
+\mathbb{E}\left[\int_{0}^{T \wedge \theta^{n}} e^{-\beta t} \mathbf{g}^{\mathbf{b}} \cdot D \varphi\left(S_{t}^{n}\right) d L_{t}^{n c}\right] \\
+\mathbb{E}\left[\int_{0}^{T \wedge \theta^{n}} e^{-\beta t}\left[(1-\alpha) Y_{t}^{n}+\alpha f^{\varepsilon}\left(S_{t}^{n}\right) K_{t}^{n}\right]\left(\mathbf{g}_{\varepsilon}^{\mathbf{s}} \cdot D \varphi\right)\left(S_{t}^{n}\right) d M_{t}^{n c}\right] \\
+\mathbb{E}\left[\sum_{0 \leq t<T \wedge \theta^{n}} e^{-\beta t}\left(\varphi\left(S_{t-}^{n}\right)-\varphi\left(S_{t}^{n}\right)\right)\right]
\end{aligned}
$$

where $L^{n c}$ and $M^{n c}$ denote the continuous part of $L^{n}$ and $M^{n}$. Recall that $\varphi$ satisfies 
(5.12), (5.13) and (5.14), then it follows from the previous inequality

$$
\begin{aligned}
& 2 \xi_{n} \geq \gamma e^{-\beta T} \mathbb{E} {\left[\left(T \wedge \theta^{n}\right)+L_{T \wedge \theta^{n}}^{n c}+\int_{0}^{T \wedge \theta^{n}}\left[(1-\alpha) Y_{t}^{n}+\alpha f^{\varepsilon}\left(S_{t}^{n}\right) K_{t}^{n}\right] d M_{t}^{n c}\right] } \\
&+\gamma e^{-\beta T} \mathbb{E}\left[\sum_{0 \leq t<T \wedge \theta^{n}} \Delta L_{t}^{n}+\left[(1-\alpha) Y_{t-}^{n}+\alpha f^{\varepsilon}\left(S_{t-}^{n}\right) K_{t-}^{n}\right] \Delta M_{t}^{n}\right] \\
&+e^{-\beta T} \gamma \mathbb{E}\left[\int_{0}^{T \wedge \theta^{n}}\left(C_{t}^{n}-c_{0}\right)^{+} d t\right], \\
& \geq \mathbb{E}\left[h^{n}\left(T \wedge \theta^{n}\right)\right],
\end{aligned}
$$

where

$$
\begin{aligned}
& h^{n}\left(T \wedge \theta^{n}\right)=\gamma e^{-\beta T}\left\{\left(T \wedge \theta^{n}\right)+L_{T \wedge \theta^{n}}^{n}\right. \\
&\left.+\int_{0}^{T \wedge \theta^{n}}\left[(1-\alpha) Y_{t-}^{n}+\alpha f^{\varepsilon}\left(S_{t-}^{n}\right) K_{t-}^{n}\right] d M_{t}^{n}+\int_{0}^{T \wedge \theta^{n}}\left(C_{t}^{n}-c_{0}\right)^{+} d t\right\} .
\end{aligned}
$$

3. In order to obtain a contradiction, we are going to show that for a sufficiently small $T$, there is some constant $m_{*}$ such that for large $n \geq 1, \mathbb{E}\left[h^{n}\left(T \wedge \theta^{n}\right)\right] \geq m_{*}$. The following argument is largely inspired from [22].

3.1. We start by providing estimates for $\left|X^{n}-x_{0}\right|,\left|Y^{n}-y_{0}\right|$, and $\left|K^{n}-k_{0}\right|$. Fix some $n \geq 1$, and assume that $n$ is sufficiently large so that $\xi_{n} \leq \eta / 2$ holds. Let $\Lambda$ be the process defined by : $\Lambda_{t}:=\left(\rho-\frac{\sigma^{2}}{2}\right) t+\sigma W_{t}$, and set

$$
\Lambda_{t}^{\star}:=\left|\rho-\frac{\sigma^{2}}{2}\right| t+\sigma\left(W_{t}^{\star}-W_{\star t}\right), \text { where } W_{t}^{\star}:=\max _{u \in[0, t]} W_{u} \text { and } W_{\star t}:=\min _{u \in[0, t]} W_{u} .
$$

Since $d\left[Y_{t}^{n} e^{-\Lambda_{t}}\right]=e^{-\Lambda_{t}} d L_{t}^{n}-e^{-\Lambda_{t}} Y_{t-}^{n} d M_{t}^{n}$, we deduce by a direct calculation that

$$
\left|Y_{t}^{n}-y_{0}\right| \leq\left|y_{0}-y_{n}\right|+y_{n}\left|1-e^{\Lambda_{t}}\right|+e^{\Lambda_{t}^{\star}} L_{t}^{n}+e^{\Lambda_{t}^{\star}} \int_{0}^{t} Y_{u-}^{n} d M_{u}^{n}
$$

The dynamics of the processes $K^{n}$ and $X^{n}$ are such that

$$
\begin{gathered}
\left|K_{t}^{n}-k_{0}\right| \leq\left|k_{0}-k_{n}\right|+L_{t}^{n}+\int_{0}^{t} f^{\varepsilon}\left(S_{u-}^{n}\right) K_{u-}^{n} d M_{u}^{n} \\
\left|X_{t}^{n}-x_{0}\right| \leq\left|x_{n}-x_{0}\right|+\left|x_{n}\right|\left(e^{r t}-1\right)+e^{r t} \int_{0}^{t} e^{-r u} C_{u}^{n} d u+e^{r t}(1+\lambda) \int_{0}^{t} e^{-r u} d L_{u}^{n} \\
\quad+e^{r t} \int_{0}^{t} e^{-r u}(1-\mu)\left[(1-\alpha) Y_{u-}^{n}+\alpha f^{\varepsilon}\left(S_{u-}^{n}\right) K_{u-}^{n}\right] d M_{u}^{n}
\end{gathered}
$$

3.2. We have $\left|1-e^{\Lambda_{T}}\right| \leq \max \left[e^{\Lambda_{T}^{\star}}-1 ; 1-e^{-\Lambda_{T}^{\star}}\right]$. Define the set

$$
F_{T}:=\left\{\omega \in \Omega: \max \left[e^{\Lambda_{T}^{\star}}-1 ; 1-e^{-\Lambda_{T}^{\star}}\right] \leq \min \left[1, \frac{\eta}{4\left(y_{0}+1\right)}\right]\right\} .
$$

We claim that it is possible to choose the parameter $T>0$ such that

$$
\mathbb{P}\left(F_{T}\right) \geq \frac{1}{2}, e^{r T}-1 \leq \frac{\eta}{4\left(1+\left|x_{0}\right|\right)}, \text { and } e^{r T} \leq 2
$$


Indeed, Doob's maximal martingale inequalities provide, for $\delta>0$

$$
\mathbb{P}\left\{W_{T}^{\star} \geq \delta\right\} \leq \frac{1}{\delta^{2}} \mathbb{E}\left[W_{T}^{\star}\right]^{2} \leq \frac{4}{\delta^{2}} \mathbb{E}\left[W_{T}\right]^{2}=\frac{4 T}{\delta^{2}}, \text { similarly } \mathbb{P}\left\{W_{\star T} \leq \delta\right\} \leq \frac{4 T}{\delta^{2}}
$$

Hence for all $\delta>0$

$$
\mathbb{P}\left\{W_{T}^{\star}-W_{\star T} \geq \delta\right\} \leq \mathbb{P}\left\{W_{T}^{\star} \geq \delta / 2\right\}+\mathbb{P}\left\{W_{\star T} \leq \delta / 2\right\} \leq \frac{32 T}{\delta^{2}} .
$$

We now turn back to the estimates (5.15)-(5.16)-(5.17) and recall that $\xi_{n} \leq \eta / 2$. Since $T$ satisfies (5.18), the following inequalities (where $A$ denotes some positive constant depending on $\left.\left(x_{0}, y_{0}, k_{0}\right)\right)$ do hold $\mathbb{P}-$ a.s. on the set $F_{T}$

$$
\begin{aligned}
& \left|X_{T}^{n}-x_{0}\right| \leq \eta / 2+\eta / 4+2 \int_{0}^{T} C_{t}^{n} d t+A L_{T}^{n}+A \int_{0}^{T} G^{\varepsilon}\left(S_{t-}^{n}\right) d M_{t}^{n}, \\
& \left|Y_{T}^{n}-y_{0}\right| \leq \eta / 2+\eta / 4+A L_{T}^{n}+A \int_{0}^{T} G^{\varepsilon}\left(S_{t-}^{n}\right) d M_{t}^{n} \\
& \left|K_{T}^{n}-k_{0}\right| \leq \eta / 2+A L_{T}^{n}+A \int_{0}^{T} G^{\varepsilon}\left(S_{t-}^{n}\right) d M_{t}^{n} .
\end{aligned}
$$

where

$$
G^{\varepsilon}(s):=(1-\alpha) y+\alpha f^{\varepsilon}(s) k \text { for } s=(x, y, k) \in \overline{\mathcal{S}} .
$$

3.3. For $\omega$ in $F_{T}$, we consider the following cases

Case 1: $\theta^{n}(\omega) \geq T$. Then, by the definition of $h^{n}\left(T \wedge \theta^{n}\right)$, we have $h^{n}\left(T \wedge \theta^{n}\right) \geq$ $\gamma e^{-\beta T} T$.

Case 2: $\theta^{n}(\omega)<T$. Recall that $S^{n}$ is càdlag̀, then, by the definition of the stopping time $\theta^{n}$, this happens when $S_{\theta^{n}}^{n}(w) \notin B\left(s_{0}, \eta\right]$, i.e.

$$
\max \left[\left|X_{\theta^{n}(\omega)}^{n}(\omega)-x_{0}\right| ;\left|Y_{\theta^{n}(\omega)}^{n}(\omega)-y_{0}\right| ;\left|K_{\theta^{n}(\omega)}^{n}(\omega)-k_{0}\right|\right] \geq \eta .
$$

Subcase 2.1: $\left|X_{\theta^{n}(\omega)}^{n}(\omega)-x_{0}\right| \geq \eta$. It follows from (5.19) that at least one of the following inequalities holds

$$
\text { (i) } \int_{0}^{\theta^{n}(\omega)} C_{t}^{n} d t \geq \eta / 16 \text { or(ii) } L_{\theta^{n}}^{n}+\int_{0}^{\theta^{n}(\omega)} G^{\varepsilon}\left(S_{t-}^{n}\right) d M_{t}^{n} \geq \frac{\eta}{8 A} \text {. }
$$

In the former case (i) :

$$
\frac{\eta}{16} \leq \int_{0}^{\theta^{n}(\omega)} C_{t}^{n} d t \leq c_{0} T+\int_{0}^{\theta^{n}(\omega)}\left(C_{t}^{n}-c_{0}\right) d t
$$

Since it is possible to choose $T$ such that $c_{0} T \leq \frac{\eta}{32}$, it follows that

$$
\frac{\eta}{16} \leq \frac{\eta}{32}+\int_{0}^{\theta^{n}(\omega)}\left(C_{t}^{n}-c_{0}\right)^{+} d t
$$

then $\eta / 32 \leq \int_{0}^{\theta^{n}(\omega)}\left(C_{t}^{n}-c_{0}\right)^{+} d t$ and it follows that

$$
h^{n}\left(T \wedge \theta^{n}\right) \geq \gamma e^{-\beta T} \int_{0}^{\theta^{n}(\omega)}\left(C_{t}^{n}-c_{0}\right)^{+} d t \geq \gamma e^{-\beta T} \frac{\eta}{32}
$$


In case (ii), it immediately follows $h^{n}\left(T \wedge \theta^{n}\right) \geq \gamma e^{-\beta T} \frac{\eta}{8 A}$.

Subcase 2.2: $\left|Y_{\theta^{n}(\omega)}^{n}-y_{0}\right| \geq \eta$. Then, it follows from inequality (5.20) that

$$
\frac{\eta}{4} \leq A\left(L_{\theta^{n}(\omega)}^{n}+\int_{0}^{\theta^{n}} G^{\varepsilon}\left(S_{t-}^{n}\right) d M_{t}^{n}\right)
$$

hence, $h^{n}\left(T \wedge \theta^{n}(\omega)\right) \geq \gamma e^{-\beta T} \frac{\eta}{4 A}$.

Subcase 2.3: $\left|K_{\theta^{n}(\omega)}^{n}-k_{0}\right| \geq \eta$. By inequality (5.21) we see that in this case

$$
\frac{\eta}{2} \leq A\left(L_{\theta^{n}(\omega)}^{n}+\int_{0}^{\theta^{n}} G^{\varepsilon}\left(S_{t-}^{n}\right) d M_{t}^{n}\right)
$$

hence, $h^{n}\left(T \wedge \theta^{n}(\omega)\right) \geq \gamma e^{-\beta T} \frac{\eta}{2 A}$.

From the several case discussed above, it follows that for $\mathbb{P}-$ a.e. $\omega$ in $F_{T}$ :

$$
h^{n}\left(T \wedge \theta^{n}(\omega)\right) \geq m_{\star}:=\gamma \min \left[T, \frac{\eta}{32}, \frac{\eta}{8 A}\right],
$$

and therefore, for $T$ sufficiently small and large $n$ :

$$
\mathbb{E}\left[h^{n}\left(T \wedge \theta^{n}\right)\right] \geq \mathbb{E}\left[\mathbf{1}_{F_{T}} h^{n}\left(T \wedge \theta^{n}\right)\right] \geq m_{\star} \mathbb{P}\left(F_{T}\right)=\frac{m_{\star}}{2} .
$$

REMARK 3. Let $\mathcal{A}_{0}(s)$ be the subset of $\mathcal{A}(s)$ consisting of all controls $\nu=$ $(C, L, M)$ with Lebesgue-absolutely continuous component $M$. Then, it is clear that the above derivation of the dynamic programming equation is not altered by this additional restriction. Hence the value problem of this new control problem coincides with $V_{\varepsilon}$, by the comparison result of Theorem 3.1. The same comment holds if the component $L$, or both components $L$ and $M$, are restricted to be Lebesgue-absolutely continuous.

6. The convergence result. We first derive a useful estimate.

Lemma 6.1. Let $s$ be in $\overline{\mathcal{S}}$. Then for any $\varepsilon \geq 0, \mathcal{A}^{\varepsilon}(s) \subset \mathcal{A}(s)$, and for all $\nu \in \mathcal{A}(s)$ and $t \geq 0:$

$$
0 \leq Z_{t}^{0, s, \nu}-Z_{t}^{\varepsilon, s, \nu} \leq 4 \varepsilon r Z_{T}^{0, s, \nu \star} e^{r t} \text { where } Z_{t}^{0, s, \nu \star}:=\sup _{u \in[0, t]}\left|Z_{u}^{0, s, \nu}\right| .
$$

Proof. Clearly the inclusion $\mathcal{A}^{\varepsilon}(s) \subset \mathcal{A}(s)$ follows from the inequality $Z^{0, s, \nu} \geq$ $Z^{\varepsilon, s, \nu}$

1. We first prove that $Z^{\varepsilon, s, \nu} \leq Z^{0, \varepsilon, \nu} \mathbb{P}$ - a.s.. To see this, we consider a sequence of stopping times $\left(\tau_{n}\right)_{n \geq 0}$ exhausting the jumps of the càdlàg process $M$, with $\tau_{0}=0$. The dynamics of the processes $K^{\varepsilon, s, \nu}$ and $K^{0, s, \nu}$ are such that

$$
d\left(K^{\varepsilon, s, \nu}-K^{0, s, \nu}\right)_{t}=-\left(K^{\varepsilon, s, \nu}-K^{0, s, \nu}\right)_{t-} d M_{t}+\left[1-f^{\varepsilon}\left(S_{t-}^{\varepsilon, s, \nu}\right)\right] K_{t-}^{\varepsilon, s, \nu} d M_{t} .
$$

Then, for all $n \geq 0$, we have $\mathbb{P}-$ a.s. for $t \in\left[\tau_{n}, \tau_{n+1}\right)$

$$
K_{t}^{\varepsilon, s, \nu}-K_{t}^{0, s, \nu}=e^{-\left(M_{t}^{c}-M_{\tau_{n}}^{c}\right)}\left(K_{\tau_{n}}^{\varepsilon, s, \nu}-K_{\tau_{n}}^{0, s, \nu}+\int_{\tau_{n}}^{t} e^{M_{u}^{c}-M_{\tau_{n}}^{c}}\left[1-f^{\varepsilon}\left(S_{u-}^{\varepsilon, s, \nu}\right)\right] K_{u-}^{\varepsilon, s, \nu} d M_{u}\right) .
$$


Since $1-f^{\varepsilon} \geq 0$, this implies that

$$
\begin{aligned}
K_{t}^{\varepsilon, s, \nu}-K_{t}^{0, s, \nu} \geq e^{-\left(M_{t}^{c}-M_{\tau_{n}}^{c}\right)}\left(K_{\tau_{n}}^{\varepsilon, s, \nu}-K_{\tau_{n}}^{0, s, \nu}\right) \\
=e^{-\left(M_{t}^{c}-M_{\tau_{n}}^{c}\right)}\left(\left(K_{\tau_{n}-}^{\varepsilon, s, \nu}-K_{\tau_{n}-}^{0, s, \nu}\right)\left(1-\Delta M_{\tau_{n}}\right)\right. \\
\left.\quad+\left[1-f^{\varepsilon}\left(S_{\tau_{n}-}^{\varepsilon, s, \nu}\right)\right] K_{\tau_{n}-}^{\varepsilon, s, \nu} \Delta M_{\tau_{n}}\right) \geq 0 .
\end{aligned}
$$

Clearly, $Y^{\varepsilon, s, \nu}=Y^{0, s, \nu}$. Then

$$
d\left(Z^{\varepsilon, s, \nu}-Z^{0, s, \nu}\right)_{t}=r\left(Z^{\varepsilon, s, \nu}-Z^{0, s, \nu}\right)_{t} d t-r(1-\mu) \alpha\left(K^{\varepsilon, s, \nu}-K^{0, s, \nu}\right) d t
$$

Since $Z_{0}^{\varepsilon, s, \nu}-Z_{0}^{0, s, \nu}=0$ and $K^{\varepsilon, s, \nu} \geq K^{0, s, \nu}$, this implies that

$$
Z_{t}^{\varepsilon, s, \nu}-Z_{t}^{0, s, \nu}=-r(1-\mu) \alpha e^{r t} \int_{0}^{t} e^{-r u}\left(K_{u}^{\varepsilon, s, \nu}-K_{u}^{0, s, \nu}\right) d u \leq 0
$$

2. We next prove the second inequality. Observe that $\left[1-f_{\varepsilon}(s)\right] k \leq 2 \varepsilon z$ for $s=$ $(x, y, k) \in \overline{\mathcal{S}}$, where $z:=x+(1-\mu)[(1-\alpha) y+\alpha k]$. Together with (6.1) and (6.2) this shows that, for all $n \geq 0$ and $t \in\left[\tau_{n}, \tau_{n+1}\right)$,

$$
K_{t}^{\varepsilon, s, \nu}-K_{t}^{0, s, \nu} \leq 2 \varepsilon e^{-\left(M_{t}^{c}-M_{\tau_{n}}^{c}\right)}\left(Z_{\tau_{n},}^{\varepsilon, s, n u}+\int_{\tau_{n}}^{t} e^{M_{u}^{c}-M_{\tau_{n}}^{c}} Z_{u-}^{\varepsilon, s, \nu} d M_{u}\right) .
$$

Using the increase of $M$ together with the fact that $Z^{\varepsilon, s, \nu} \leq Z^{0, s, \nu}$, as shown in the first step of this proof, this provides

$$
K_{t}^{\varepsilon, s, \nu}-K_{t}^{0, s, \nu} \leq 2 \varepsilon Z_{t}^{0, s, \nu \star} e^{-\left(M_{t}^{c}-M_{\tau_{n}}^{c}\right)}\left(1+\int_{\tau_{n}}^{t} e^{M_{u}^{c}-M_{\tau_{n}}^{c}} d M_{u}\right) \leq 4 \varepsilon Z_{t}^{0, s, \nu \star} .
$$

The required inequality is obtained by plugging this estimate into (6.3).

Proposition 6.2. The sequence $\left(V_{\varepsilon}\right)_{\varepsilon>0}$ is non-increasing and $V_{\varepsilon} \leq V$.

Proof. The inequality $V_{\varepsilon} \leq V$ follows immediately from the fact that $\mathcal{A}^{\varepsilon}(s) \subset \mathcal{A}(s)$, as stated in Lemma 6.1. In order to prove that the sequence $\left(V_{\varepsilon}\right)_{\varepsilon>0}$ is non-increasing, we shall prove that $\mathcal{A}^{\varepsilon_{1}}(s) \subset \mathcal{A}^{\varepsilon_{2}}(s)$ whenever $\varepsilon_{1} \geq \varepsilon_{2}$. To do this, it is sufficient to prove that for any control $\nu=(C, L, M) \in \mathcal{A}_{\varepsilon}(s)$, the associated process $Z^{\varepsilon}:=$ $X^{\varepsilon, s, \nu}+(1-\mu)\left[(1-\alpha) Y^{\varepsilon, s, \nu}+\alpha K^{\varepsilon, s, \nu}\right]$ is non-increasing with respect to $\varepsilon$. Recall that

$$
f^{\varepsilon}(s)=h\left(\frac{k}{\varepsilon z}\right) \text { where } z=x+(1-\mu)[(1-\alpha y+\alpha z],
$$

and $h$ is a smooth function. From Remark 3, we may restrict the process $M$ to be absolutely continuous with respect to the Lebesgue measure, i.e. $M_{t}=\int_{0}^{t} m_{u} d u$ for some $\mathbb{F}$-adapted process $\left\{m_{t}, t \geq 0\right\}$, as the restriction of the control $M$ to this class produces the same value function $V_{\varepsilon}$.

Then, by classical results on the regularity of flows of stochastic differential equations, see e.g. [16], the process $Z^{\varepsilon}, Y^{\varepsilon}:=Y^{\varepsilon, s, \nu}$ and $K^{\varepsilon}:=K^{\varepsilon, s, \nu}$ are differentiable in $\varepsilon$, and the processes

$$
z_{t}^{\varepsilon}:=e^{-r t} \frac{\partial Z_{t}^{\varepsilon}}{\partial \varepsilon}, y_{t}^{\varepsilon}:=\frac{\partial Y_{t}^{\varepsilon}}{\partial \varepsilon}, k_{t}^{\varepsilon}:=e^{-r t} \frac{\partial K_{t}^{\varepsilon}}{\partial \varepsilon}
$$


satisfy $y_{t}^{\varepsilon}=0$ for all $t \geq 0, z_{0}^{\varepsilon}=k_{0}^{\varepsilon}=0$, and solve the system of ordinary differential equations

$$
\dot{z}_{t}^{\varepsilon}=-r \alpha k_{t} \text { and } \dot{k}_{t}^{\varepsilon}=a_{t}+b_{t} z_{t}-c_{t} k_{t}
$$

where

$$
a_{t}:=\frac{\left(K_{t}^{\varepsilon}\right)^{2}}{\varepsilon Z_{t}^{\varepsilon}} h^{\prime}\left(\frac{K_{t}^{\varepsilon}}{\varepsilon Z_{t}^{\varepsilon}}\right), b_{t}:=\frac{\left(K_{t}^{\varepsilon}\right)^{2}}{\varepsilon\left(Z_{t}^{\varepsilon}\right)^{2}} h^{\prime}\left(\frac{K_{t}^{\varepsilon}}{\varepsilon Z_{t}^{\varepsilon}}\right)
$$

and

$$
e^{-r t} c_{t}:=r+m_{t}\left[h\left(\frac{K_{t}^{\varepsilon}}{\varepsilon Z_{t}^{\varepsilon}}\right)+\frac{K_{t}^{\varepsilon}}{\left.\varepsilon Z_{t}^{\varepsilon}\right)} h^{\prime}\left(\frac{K_{t}^{\varepsilon}}{\varepsilon Z_{t}^{\varepsilon}}\right)\right] .
$$

Differentiating once more with respect to the $t$-variable, we obtain the following second order differential equation for $z^{\varepsilon}$ :

$$
-\ddot{z}_{t}^{\varepsilon}-c_{t} \dot{z}_{t}^{\varepsilon}-r \alpha b_{t} z_{t}^{\varepsilon}-r \alpha a_{t}=0 \text { and } \dot{z}_{0}^{\varepsilon}=z_{0}=0 .
$$

We now consider the function :

$$
\hat{z}_{t}:=-\frac{r \alpha}{\varepsilon} \int_{0}^{t} \int_{0}^{u} \frac{\left(K_{t}^{\varepsilon}\right)^{2}}{\varepsilon Z_{t}^{\varepsilon}} h^{\prime}\left(\frac{K_{t}^{\varepsilon}}{\varepsilon Z_{t}^{\varepsilon}}\right) d u d t \text { for } t \geq 0 .
$$

Since $\hat{z}_{t} \leq 0, \dot{\hat{z}}_{t} \leq 0, b_{t} \geq 0$ and $c \geq 0$, it follows that $\hat{z}_{t}$ is a supersolution of the equation (6.4). By a standard comparison result, we deduce that $z_{t}^{\varepsilon} \leq \hat{z}_{t}$, and therefore $z_{t}^{\varepsilon} \leq 0$ for all $t \geq 0$. This completes the proof.

Our final result states the convergence of $V_{\varepsilon}$ towards $V$.

Proposition 6.3. The sequence $\left(V_{\varepsilon}\right)_{\varepsilon>0}$ is non-increasing and converges towards $V$, as $\varepsilon \searrow 0$, uniformly on compact subsets of $\overline{\mathcal{S}}$

Proof. Let $\left(\nu^{n}=\left(C^{n}, L^{n}, M^{n}\right)\right)_{n \geq 1}$ be a maximizing sequence of controls for $V(s)$ :

$$
V(s)-\frac{1}{n} \leq \mathbb{E}\left[\int_{0}^{\infty} e^{-\beta t} U\left(C_{t}^{n}\right) d t\right] \text { for all } n \geq 1
$$

By the monotone convergence theorem we verify that

$$
\mathbb{E}\left[\int_{0}^{\infty} e^{-\beta t} U\left(C_{t}^{n}\right) d t\right]=\lim _{T \rightarrow \infty} \mathbb{E}\left[\int_{0}^{T} e^{-\beta t} U\left(C_{t}^{n}\right) d t\right] .
$$

Then $V(s)-\frac{1}{2 n} \leq \mathbb{E}\left[\int_{0}^{T^{n}} e^{-\beta t} U\left(C_{t}^{n}\right) d t\right]$ for some $T^{n}>0$. By Lemma 6.1we have $Z_{t \wedge T^{n}}^{0, s, \nu} \geq Z_{t \wedge T^{n}}^{\varepsilon, s, \nu} \geq Z_{t \wedge T^{n}}^{0, s, \nu}-4 r \varepsilon Z_{T^{n}}^{0, s, \nu \star} \mathbb{P}-$ a.s. for all $t \geq 0$. Then, the stopping times $\tau(\varepsilon, s, n):=\inf \left\{t \geq 0: Z_{t}^{\varepsilon, s, \nu} \leq 0\right\}, \varepsilon \geq 0$, satisfy

$$
\tau(0, s, n) \wedge T^{n} \geq \tau(\varepsilon, s, n) \wedge T^{n} \text { and } \lim _{\varepsilon \rightarrow 0} \tau(\varepsilon, s, n) \wedge T^{n}=\tau(0, s, n) \wedge T^{n} \mathbb{P}-\text { a.s. }
$$

Hence, by the monotone convergence theorem

$$
\lim _{\varepsilon \rightarrow 0} \mathbb{E}\left[\int_{0}^{\tau(\varepsilon, s, n) \wedge T^{n}} e^{-\beta t} U\left(C_{t}^{n}\right) d t\right]=\mathbb{E}\left[\int_{0}^{\tau(0, s, n) \wedge T^{n}} e^{-\beta u} U\left(C_{t}^{n}\right) d t\right]
$$


Recall from (5.6) and (5.7) that

$$
\begin{aligned}
V_{\varepsilon}(s) \geq \mathbb{E} & {\left[\int_{0}^{\tau(\varepsilon, s, n) \wedge T^{n}} e^{-\beta t} U\left(C_{t}^{n}\right) d t\right] \text { and } } \\
& \mathbb{E}\left[\int_{0}^{\tau(0, s, n) \wedge T^{n}} e^{-\beta t} U\left(C_{t}^{n}\right) d t\right]=\mathbb{E}\left[\int_{0}^{T^{n}} e^{-\beta u} U\left(C_{u}^{n}\right) d u\right] .
\end{aligned}
$$

Then

$$
\liminf _{\varepsilon \rightarrow 0} V_{\varepsilon}(s) \geq \mathbb{E}\left[\int_{0}^{T^{n}} e^{-\beta t} U\left(C_{t}^{n}\right) d t\right] \geq V(s)-\frac{1}{2 n} .
$$

By arbitrariness of $n \geq 1$, this provides $\liminf _{\varepsilon \rightarrow 0} V_{\varepsilon}(s) \geq V(s)$. Together with Proposition 6.2, this shows that $V_{\varepsilon}(s) \longrightarrow V(s)$ as $\varepsilon \searrow 0$ for every $s \in \overline{\mathcal{S}}$.

We finally recall from Proposition 2.4 that the limit function $V$ is continuous. Since $\left(V_{\varepsilon}\right)_{\varepsilon>0}$ is a monotonic sequence of continuous functions, it follows from the Dini theorem that the convergence holds uniformly on compact subsets of $\overline{\mathcal{S}}$.

\section{REFERENCES}

[1] M. Akian, J.L. Menaldi and A. Sulem (1996) On an investment-consumption model with transaction costs, SIAM Journal on control and Optimization 34, 329-364.

[2] G. Barles and J. Burdeau (1995). The Dirichlet problem for semilinear second-order degenerate elliptic equations and applications to stochastic exit time control problems. Communications in Partial Differential Equations 20, 129-178.

[3] G. Barles and E. Rouy (1998). A strong comparison result for the Bellman equation arising in stochastic exit time control problems and its applications. Communications in Partial Differential Equations 23, 1945-2033.

[4] G. Barles and P.E. Souganidis (1991). Convergence of approximation schemes for fully nonlinear equations. Asymptotic Analysis 4, 271-283.

[5] Ben Tahar I, M. Soner and N. Touzi (2005). Modeling continuous-time financial markets with capital gains taxes. Preprint.

[6] Borkar, V.S. (1989). Optimal Control of Diffusion Processes. Pitman Research Notes, New York.

[7] G.M. Constantinides (1983) Capital market equilibrium with personal taxes, Econometrica 51, 611-636.

[8] J. Cox and C.F. Huang (1989). Optimal consumption and portfolio policies when asset prices follow a diffusion process, Journal of Economic Theory 49, 33-83.

[9] M.G. Crandall, H. Ishii and P.L. Lions (1992). User's guide to viscosity solutions of second order partial differential equations, Bullein (New series) of the American Mathematical Society 27.

[10] R.M. Dammon, C.S. Spatt and H.H. Zhang (2001). Optimal consumption and investment with capital gains taxes. The Review of Financial Studies 14, 583-616.

[11] V. DeMiguel and R. Uppal (2005). Portfolio investment with the exact tax basis via nonlinear programming. Management Science 51, 277-290.

[12] P. Dybvig and H. Koo (1996). Investment with taxes. Working paper, Washington University, Saint Louis, MO.

[13] El Karoui, N. (1981). Les aspects probabilistes du contrôle stochastique. Lecture Notes in Mathematics 876, 513-537. Springer-Verlag.

[14] Fleming, W.H., Soner, H.M. (1993). Controlled Markov Processes and Viscosity Solutions. Applications of Mathematics 25. Springer-Verlag, New York.

[15] M. Gallmeyer, R. Kaniel and S. Tompaidis (2002). Tax Management Strategies with Multiple Risky Assets, preprint.

[16] Ikeda, N., Watanabe, S. (1989). Stochastic Differential Equations and Diffusion Processes, Second Edition. North-Holland Publishing Company.

[17] E. Jouini, P.-F. Koehl and N. Touzi (1997). Optimal investment with taxes : an optimal control problem with endogeneous delay, Nonlinear Analysis : Theory, Methods and Applications 37, 31-56. 
[18] E. Jouini, P.-F. Koehl and N. Touzi (1999). Optimal investment with taxes: an existence result, Journal of Mathematical Economics 33, 373-388.

[19] Leland, H.E. (1999). Optimal Portfolio Management with Transactions Costs and Capital Gains Taxes. Preprint.

[20] R.C. Merton (1969). Lifetime portfolio selection under uncertainty: the continuous-time model. Review of Economic Statistics 51, 247-257.

[21] R.C. Merton (1971). Optimum consumption and portfolio rules in a continuous-time model. Journal of Economic Theory 3, 373-413.

[22] S.E. Shreve, H.M. Soner (1994). Optimal investment and consumption with transaction costs, Annals of Applied Probability 4, 609-692.

[23] H.M. Soner (1986). Optimal control with state space constraint I, SIAM J. Control Optim., 24, 552-562 ; II, 24 (1986) 1110-1122. 